\title{
The algebraic crossing number and the braid index of knots and links
}

\author{
KeIKO KAWAMURO
}

\begin{abstract}
It has been conjectured that the algebraic crossing number of a link is uniquely determined in minimal braid representation. This conjecture is true for many classes of knots and links.

The Morton-Franks-Williams inequality gives a lower bound for braid index. And sharpness of the inequality on a knot type implies the truth of the conjecture for the knot type.

We prove that there are infinitely many examples of knots and links for which the inequality is not sharp but the conjecture is still true. We also show that if the conjecture is true for $\mathcal{K}$ and $\mathcal{L}$, then it is also true for the $(p, q)$-cable of $\mathcal{K}$ and for the connect sum of $\mathcal{K}$ and $\mathcal{L}$.
\end{abstract}

57M25; 57M27

\section{Introduction}

The braid index is one of the classical invariants of knots and links. Any knot and link type is presented as a braid closure. The braid index of a link type is the least number of braid strands needed for that.

The algebraic crossing number (or writhe) is an integer associated to an oriented link diagram counting the crossings with weight +1 (resp. -1 ) for a positive (resp. negative) crossing as shown in the left (resp. middle) sketch of Figure 1. Since it is changed under Reidemeister move I, it is not an invariant of link types. However, it has been asked (see Jones [8, page 357] for example):

Question Is the algebraic crossing number in a minimal braid representation a link invariant?

Here "minimal" means that the number of braid strands of a link diagram is equal to the braid index of the link type. 
It is known that the following links have unique algebraic crossing numbers at minimal braid index: torus links, closed positive braids with a full twist, including the Lorenz links (Franks and Williams [6]), 2-bridge links and alternating fibered links (Murasugi [14]) and links with braid index $\leq 3$ (Birman and Menasco [4]).

In Section 3 of this paper we approach the above question in three ways. The first way (Theorem 3.5 and its corollaries) is by studying the deficit of the Morton-FranksWilliams (MFW) inequality (Morton [12], Franks and Williams [6]). It is easy to see that sharpness of the MFW-inequality implies the uniqueness of the algebraic crossing number at minimal index. Then how do we answer the question for links on which the inequality is not sharp? In fact we provide infinitely many examples of non-sharp links having unique algebraic crossing numbers at minimal braid index.

The second way is by studying the behavior of the braid index and the algebraic crossing number under the cabling operation. In Theorem 3.10 and Theorem 3.12, we will prove that the uniqueness property is preserved under cabling. Then we have Corollary 3.15 saying "yes" to the question for iterated torus knots.

The third way is by studying the connect sum operation. In Theorem 3.16, we will show that the uniqueness property is preserved under taking the connect sum.

In Section 2, we focus on non-sharpness of the MFW-inequality.

To state the MFW-inequality, let $\mathcal{K}$ be an oriented knot type and let $K$ be a diagram of $\mathcal{K}$ on a plane. Focus on one crossing of $K$ with sign $\varepsilon$. Denote $K_{\varepsilon}:=K$ and let $K_{-\varepsilon}$ (resp. $K_{0}$ ) be the closed braid obtained from $K_{\varepsilon}$ by changing the the crossing to the opposite sign $-\varepsilon$ (resp. resolving the crossing), see Figure 1.
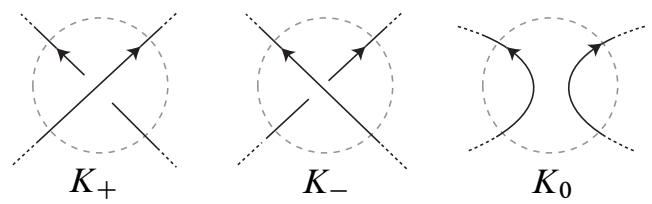

Figure 1: Local views of $K_{+}, K_{-}, K_{0}$

The HOMFLYPT polynomial $P_{\mathcal{K}}(v, z)=P_{K}(v, z)$ satisfies the following relations (for any choice of a crossing):

$$
\begin{aligned}
\frac{1}{v} P_{K_{+}}-v P_{K_{-}} & =z P_{K_{0}} . \\
P_{\text {unknot }} & =1 .
\end{aligned}
$$

Now we are ready to state the MFW-inequality. 
Theorem 1.1 (The Morton-Franks-Williams inequality [12;6]) Let $d_{+}$and $d_{-}$be the maximal and minimal degrees of the variable $v$ of $P_{\mathcal{K}}(v, z)$. If a knot type $\mathcal{K}$ has a closed braid representative $K$ with braid index $b_{K}$ and algebraic crossing number $c_{K}$, then we have

$$
c_{K}-b_{K}+1 \leq d_{-} \leq d_{+} \leq c_{K}+b_{K}-1 \text {. }
$$

As a corollary,

$$
\frac{1}{2}\left(d_{+}-d_{-}\right)+1 \leq b_{K}
$$

giving a lower bound for the braid index $b_{\mathcal{K}}$ of $\mathcal{K}$.

In general, it is hard to determine the braid index. This inequality was the first known result of a general nature relating to the computation of braid index, and it appeared to be quite effective. Jones notes, in [8], that on all but five knots, $9_{42}, 9_{49}, 10_{132}, 10_{150}, 10_{156}$ in the standard knot table, up to crossing number 10 , the MFW inequality is sharp. Furthermore it has been known that the inequality is sharp on all torus links, closed positive $n$-braids with a full twist [6], 2-bridge links and fibered alternating links [14].

However, the MFW-inequality is not as strong as it appears to be as above. In Theorem 2.5 we give an infinite class of prime links in which the deficit $D_{\mathcal{K}}:=b_{\mathcal{K}}-\frac{1}{2}\left(d_{+}-d_{-}\right)-1$ of the MFW-inequality (3) can be arbitrarily large. And in Theorem 2.8 we see another infinite class of knots, including $9_{42}, 9_{49}, 10_{132}, 10_{150}, 10_{156}$, on which the inequality is not sharp.

Then we may ask "why does non-sharpness occur?" Theorem 2.2 gives a sufficient condition for non-sharpness of the MFW inequality. In fact all the examples in Theorems 2.5 and 2.8 satisfy this sufficient condition.

The idea of Theorem 2.2 is to find knots $K_{\alpha}$ of known braid index $=b$ which have a distinguished crossing such that, after changing that crossing to each of the other two possibilities in Figure 1, giving knots or links $K_{\beta}$ and $K_{\gamma}$, it is revealed that $K_{\beta}$ and $K_{\gamma}$ each has braid index $<b$.

Thanks to Theorem 2.2 one can visually observe the "accumulation" of deficits (for example under the connect sum operation and other linking operation) by looking only at the distinguished crossings which contribute to deficits. See the proof of Theorem 2.5 for details.

Acknowledgment This paper is part of the author's $\mathrm{PhD}$ thesis. She is grateful to her advisor, Joan Birman, for her thoughtful advice and encouragement. She also wishes 
to thank William Menasco, who told her about the Birman-Menasco diagram and the associated conjecture, when she visited SUNY Buffalo. She appreciates many helpful comments by Walter Neumann, Dylan Thurston, Ilya Kofman and the referee and thanks Alexander Stoimenow for sending a preprint She acknowledges partial support from NSF grants DMS-0405586 and DMS-0306062. Finally, she especially thanks Mikami Hirasawa, who shared many creative ideas and results about fibered knots including the definition and properties of the enhanced Milnor number.

\section{Non-sharpness of the Morton-Franks-Williams inequality}

\subsection{Sufficient conditions for non-sharpness}

We define the deficit of MFW-inequality (Definition 2.1) then give sufficient conditions (Theorem 2.2) for a closed braid on which the inequality is not sharp.

Let $b_{\mathcal{K}}$ be the braid index of knot type $\mathcal{K}$, that is the smallest integer $b_{\mathcal{K}}$ such that $\mathcal{K}$ can be represented by a closed $b_{\mathcal{K}}$-braid. Let $b_{K}, c_{K}$ denote the braid index and the algebraic crossing number of a braid representative $K$ of $\mathcal{K}$.

Definition 2.1 Let

$$
D_{\mathcal{K}}:=b_{\mathcal{K}}-\frac{1}{2}\left(d_{+}-d_{-}\right)-1
$$

be the difference of the numbers in (3), ie, of the actual braid index and the lower bound for braid index. Call $D_{\mathcal{K}}$ the deficit of the MFW-inequality for $\mathcal{K}$.

If $D_{\mathcal{K}}=0$, the MFW-inequality is sharp on $\mathcal{K}$. If $K$ is a braid representative of $\mathcal{K}$ let $D_{K}^{+}:=\left(c_{K}+b_{K}-1\right)-d_{+}$and $D_{K}^{-}:=d_{-}-\left(c_{K}-b_{K}+1\right)$. When $b_{K}=b_{\mathcal{K}}$, we have

$$
D_{\mathcal{K}}=\frac{1}{2}\left(D_{K}^{+}+D_{K}^{-}\right)
$$

Note that $D_{K}^{ \pm}$depends on the choice of braid representative $K$, but the deficit $D_{\mathcal{K}}$ is independent from the choice.

Theorem 2.2 Assume that $K$ is a closed braid representative of $\mathcal{K}$ with $b_{K}=b_{\mathcal{K}}$. Focus on one site of $K$ and construct $K_{+}, K_{-}, K_{0}$ (one of the three must be $K$ ). Let $\alpha, \beta, \gamma \in\{+,-, 0\}$ and assume that $\alpha, \beta, \gamma$ are mutually distinct. If $K_{\alpha}=K$ and positive destabilization is applicable $p$-times to each of $K_{\beta}$ and $K_{\gamma}$, then

$$
D_{K}^{+} \geq 2 p ;
$$

Algebraic 83 Geometric Topology, Volume 6 (2006) 
and if $K_{\alpha}=K$ and negative destabilization is applicable $n$-times to each of $K_{\beta}$ and $K_{\gamma}$, then

$$
D_{K}^{-} \geq 2 n
$$

Therefore, by (4), the MFW-inequality is not sharp on $\mathcal{K}$ if $p+n>0$.

Here is a lemma to prove Theorem 2.2.

Lemma 2.3 Let $K$ be a closed braid. Choose one crossing, and construct $K_{+}, K_{-}, K_{0}$ (one of the three must be $K$ ). We have

$$
\begin{aligned}
& d_{+}\left(P_{K_{+}}\right) \leq \max \left\{d_{+}\left(P_{K_{-}}\right)+2, \quad d_{+}\left(P_{K_{0}}\right)+1\right\} \\
& d_{+}\left(P_{K_{-}}\right) \leq \max \left\{d_{+}\left(P_{K_{+}}\right)-2, \quad d_{+}\left(P_{K_{0}}\right)-1\right\} \\
& d_{+}\left(P_{K_{0}}\right) \leq \max \left\{d_{+}\left(P_{K_{+}}\right)-1, \quad d_{+}\left(P_{K_{-}}\right)+1\right\}
\end{aligned}
$$

and

$$
\begin{array}{ll}
d_{-}\left(P_{K_{+}}\right) \geq \min \left\{d_{-}\left(P_{K_{-}}\right)+2,\right. & \left.d_{-}\left(P_{K_{0}}\right)+1\right\} \\
d_{-}\left(P_{K_{-}}\right) \geq \min \left\{d_{-}\left(P_{K_{+}}\right)-2,\right. & \left.d_{-}\left(P_{K_{0}}\right)-1\right\} \\
d_{-}\left(P_{K_{0}}\right) \geq \min \left\{d_{-}\left(P_{K_{+}}\right)-1,\right. & \left.d_{-}\left(P_{K_{-}}\right)+1\right\}
\end{array}
$$

Proof of Lemma 2.3 By (1), we have $P_{K_{+}}=v^{2} P_{K_{-}}+v z P_{K_{0}}$. Thus, $d_{+}\left(P_{K_{+}}\right)=$ $d_{+}\left(v^{2} P_{K_{-}}+v z P_{K_{0}}\right) \leq \max \left\{d_{+}\left(v^{2} P_{K_{-}}\right), d_{+}\left(v z P_{K_{0}}\right)\right\}$ and we obtain (7). The other results follow similarly.

Table (10) shows the changes of $c_{K}, b_{K}, c_{K}-b_{K}+1$ and $c_{K}+b_{K}-1$ under stabilization and destabilization of a closed braid.

\begin{tabular}{|l|c|c|c|c|}
\hline & $c_{K}$ & $b_{K}$ & $c_{K}-b_{K}+1$ & $c_{K}+b_{K}-1$ \\
\hline+ stabilization & +1 & +1 & 0 & +2 \\
\hline + destabilization & -1 & -1 & 0 & -2 \\
\hline - stabilization & -1 & +1 & -2 & 0 \\
\hline - destabilization & +1 & -1 & +2 & 0 \\
\hline
\end{tabular}

Note that $c_{K}$ and $b_{K}$ are invariant under braid isotopy and exchange moves.

Proof of Theorem 2.2 Suppose that $K=K_{\alpha}=K_{+}$. Suppose we can apply positive destabilization $k$-times $(k \geq p)$ to $K_{-}$. Let $\widetilde{K}_{-}$denote the closed braid obtained after the destabilization. Then we have:

Algebraic $8 \mathcal{G}$ Geometric Topology, Volume 6 (2006) 


$$
\begin{aligned}
d_{+}\left(P_{K_{-}}\right)+2 & =d_{+}\left(P_{\widetilde{K}_{-}}\right)+2 \\
& \leq\left(c_{\tilde{K}_{-}}+b_{\widetilde{K}_{-}}-1\right)+2 \\
& =\left\{\left(c_{K_{-}}+b_{K_{-}}-1\right)-2 k\right\}+2 \\
& =\left(c_{K_{+}}-2\right)+b_{K_{+}}-1-2 k+2 \\
& =\left(c_{K_{+}}+b_{K_{+}}-1\right)-2 k=\left(c_{K}+b_{K}-1\right)-2 k .
\end{aligned}
$$

The first equality holds since $K_{-}$and $\widetilde{K}_{-}$have the same knot type. The first inequality is the MFW-inequality. The second equality follows from Table (10).

Similarly, if we can apply positive destabilization $l$-times $(l \geq p)$ to $K_{0}$, and obtain $\tilde{K}_{0}$, we have

$$
\begin{aligned}
d_{+}\left(P_{K_{0}}\right)+1 & =d_{+}\left(P_{\tilde{K}_{0}}\right)+1 \\
& \leq\left(c_{\boldsymbol{K}_{0}}+b_{\tilde{K}_{0}}-1\right)+1 \\
& =\left(c_{K_{0}}+b_{K_{0}}-1-2 l\right)+1 \\
& =\left(c_{K_{+}}-1\right)+b_{K_{+}}-1-2 l+1 \\
& =\left(c_{K_{+}}+b_{K_{+}}-1\right)-2 l=\left(c_{K}+b_{K}-1\right)-2 l .
\end{aligned}
$$

By (7), (11) and (12) we get

$$
\begin{aligned}
d_{+}\left(P_{K}\right) & =d_{+}\left(P_{K_{+}}\right) \leq \max \left\{d_{+}\left(P_{K_{-}}\right)+2, \quad d_{+}\left(P_{K_{0}}\right)+1\right\} \\
& \leq\left(c_{K}+b_{\mathcal{K}}-1\right)-\min \{2 k, 2 l\},
\end{aligned}
$$

ie, $D_{K}^{+} \geq \min \{2 k, 2 l\} \geq 2 p$. When $K_{\alpha}=K_{-}$or $K_{\alpha}=K_{0}$, the same arguments work (use (8) or (9) for these cases in the place of (7)) and we get (5).

The other inequality (6) also holds by the identical argument.

\subsection{Deficit growth}

Our goal is to exhibit examples (Theorem 2.5) of prime links on which the deficit of the inequality can be arbitrary large.

Theorem 2.4 Knot type $\mathcal{K}=9_{42}$ has a braid representative $K=K_{+}$(see Figure 2) satisfying the sufficient condition in Theorem 2.2.

Proof of Theorem 2.4 It is known that $9_{42}$ has braid index $=4$ and deficit $D_{9_{42}}=1$. Let $K=K_{+}$be its braid representative of the minimal braid index as in Figure 2 . Construct $K_{-}, K_{0}$ by changing the shaded crossing. Sketches show that both $K_{-}, K_{0}$ can be positively destabilized. Thus by Theorem $2.2, D_{K}^{+} \geq 2$ and $D_{9_{42}} \geq 1$. 


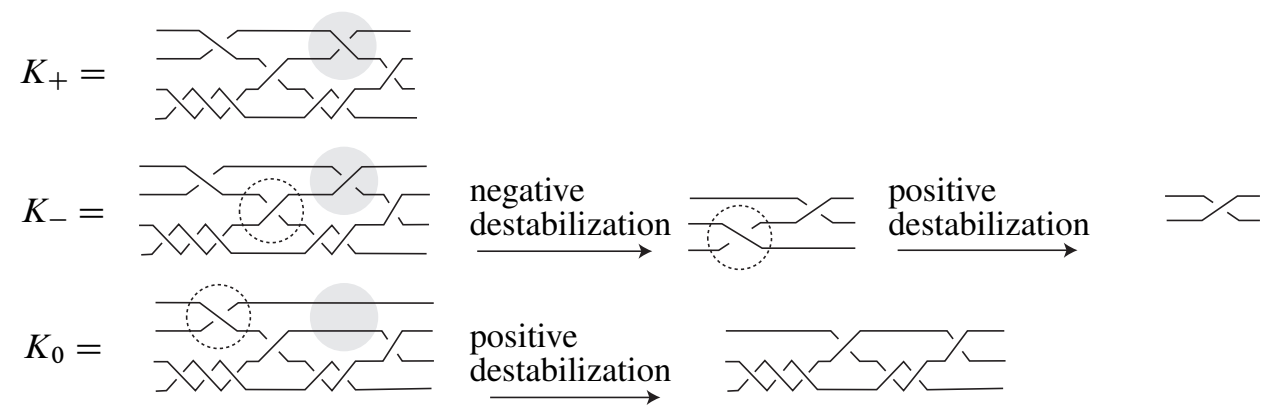

Figure 2: Knot $9_{42}$ satisfies the conditions of Theorem 2.2

Theorem 2.5 For any positive integer $n$, there exists a prime link $L$ whose deficit $D_{L} \geq n$.

Proof of Theorem 2.5 We prove the theorem by exhibiting examples. For $n \in \mathbb{N}$ let $\mathcal{A}^{n}\left(9_{42}\right)$ be the closure of $n$-copies of $9_{42}$ linked each other by two full twists as in the left sketch of Figure 3. Since the braid index $b_{9_{42}}=4$ and $\mathcal{A}^{n}\left(9_{42}\right)$ is an $n$-component link, we know the braid index of $\mathcal{A}^{n}\left(9_{42}\right)$ is $4 n$. This construction gives a braid representative with $4 n$-strands and $n$ distinguished crossings shaded in the left sketch.
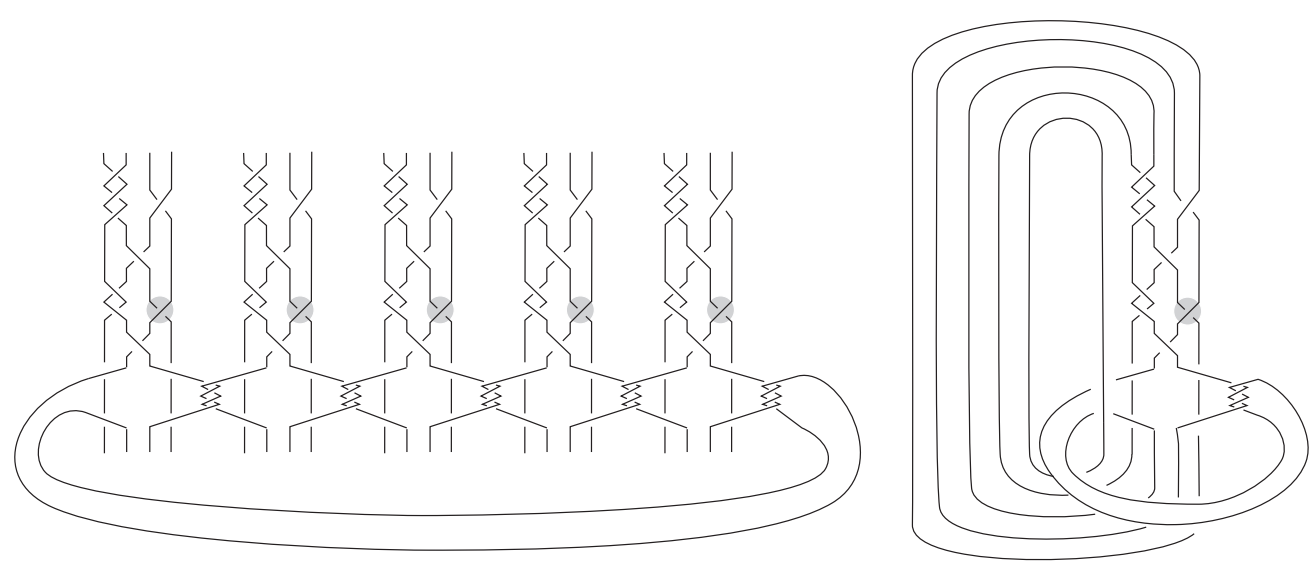

Figure 3: Prime link $\mathcal{A}^{5}\left(9_{42}\right)$ and 2-component link $\mathcal{A}$

In the following we will see that each of the shaded crossing contributes to the deficit.

Algebraic $6 \mathcal{G}$ Geometric Topology, Volume 6 (2006) 
Let $\mathcal{K}:=\mathcal{A}^{2}\left(9_{42}\right)$ and let $K$ be the braid representative of $\mathcal{K}$ as in Figure 3. Let $K_{--}, K_{-0}, K_{0-}, K_{00}$ be the links obtained from $K$ by changing the two shaded crossings. We repeat the discussion of the proof of Theorem 2.2: We have:

$$
\begin{aligned}
d_{+}\left(P_{K_{--}}\right)+(2+2) & =d_{+}\left(P_{\tilde{K}_{--}}\right)+4 \\
& \leq\left(c_{\tilde{K}_{--}}+b_{\tilde{K}_{--}}-1\right)+4 \\
& =\left\{\left(c_{K_{--}}+b_{K_{--}}-1\right)-2 \cdot 2\right\}+4 \\
& =\left(c_{K}-4\right)+b_{K}-1-2 \cdot 2+4 \\
& =\left(c_{K}+b_{K}-1\right)-2 \cdot 2
\end{aligned}
$$

Similarly:

$$
\begin{aligned}
d_{+}\left(P_{K_{-0}}\right)+(2+1) & \leq\left(c_{K}+b_{K}-1\right)-2 \cdot 2 \\
d_{+}\left(P_{K_{0-}}\right)+(1+2) & \leq\left(c_{K}+b_{K}-1\right)-2 \cdot 2 \\
d_{+}\left(P_{K_{00}}\right)+(1+1) & \leq\left(c_{K}+b_{K}-1\right)-2 \cdot 2
\end{aligned}
$$

Thus

$$
\begin{aligned}
& d_{+}\left(P_{K}\right)=\max \left\{d_{+}\left(P_{K_{--}}\right)+4, d_{+}\left(P_{K_{-0}}\right)+3, d_{+}\left(P_{K_{0-}}\right)+3, d_{+}\left(P_{K_{00}}\right)+2\right\} \\
& \leq\left(c_{K}+b_{\mathcal{K}}-1\right)-2 \cdot 2 \\
& \text { and } \quad D_{\mathcal{K}} \geq \frac{1}{2} D_{K}^{+} \geq \frac{1}{2}(2 \cdot 2)=2 \text {. }
\end{aligned}
$$

Similar arguments work when $\mathcal{K}=\mathcal{A}^{n}\left(9_{42}\right)$ for $n \geq 3$ and we have $D_{\mathcal{A}^{n}\left(9_{42}\right)} \geq$ $\frac{1}{2} D_{\mathcal{A}^{n}\left(9_{42}\right)}^{+} \geq \frac{1}{2}(2 \cdot n) \geq n$.

The 2-component link $\mathcal{A}$ of the right sketch is hyperbolic [15]. Pair $\left(S^{3}, \mathcal{A}^{n}\left(9_{42}\right) \cup\right.$ $z$-axis $)$ is an $n$-fold cover of $\left(S^{3}, \mathcal{A}\right)$ branched at $z$-axis . Therefore, by Neumann and Zagier [17] we can conclude that $\mathcal{A}^{n}\left(9_{42}\right)$ 's are all prime except for finitely many $n$ 's.

Remark 2.6 By taking the connected sum of knots on which the MFW inequality is non-sharp, one can also construct examples of (non-prime) knots with arbitrarily large deficits. This fact follows not only by Theorem 2.2 but also by the definition of HOMFLYPT polynomial (1) and the additivity of braid indices under connected sums (Birman and Menasco [3]).

\subsection{Birman-Menasco diagram}

As an application of Theorem 2.2, we study another infinite class of knots including all the Jones' five knots $\left(9_{42}, 9_{49}, 10_{132}, 10_{150}, 10_{156}\right)$ on which the MFW-inequality 
is not sharp. We call the block-strand diagram (see [5] for definition) of Figure 4 the Birman-Menasco (BM) diagram.

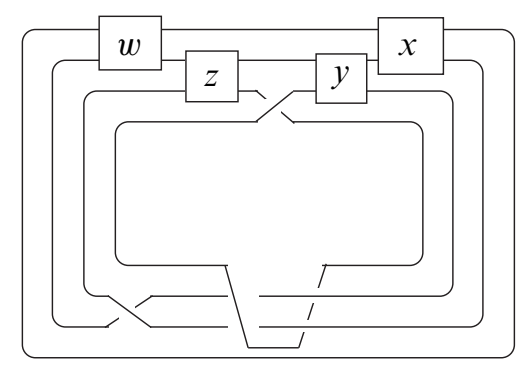

Figure 4: The Birman-Menasco diagram $B M_{x, y, z, w}$

Definition 2.7 Let $B M_{x, y, z, w}$, where $x, y, z, w \in \mathbb{Z}$, be the knot (or the link) type which is obtained by assigning $x$ (resp. $y, z, w$ ) horizontal positive half-twists on two strands to the block $X$ (resp. $Y, Z, W$ ) of the BM diagram.

Recall that on all but only five knots $\left(9_{42}, 9_{49}, 10_{132}, 10_{150}, 10_{156}\right)$ up to crossing number 10 the MFW-inequality is sharp. An interesting property of the BM diagram is that it carries all the five knots. Namely, we have $9_{42}=B M_{-1,1,-2,-1}=B M_{-1,-2,-2,2}$, $9_{49}=B M_{-1,1,1,2}, 10_{132}=B M_{-1,-2,-2,-2}, 10_{150}=B M_{3,-2,-2,2}=B M_{-1,2,-2,2}$ $=B M_{-1,-2,2,2}=B M_{-1,1,2,-1}=B M_{3,1,-2,-1}$, and $10_{156}=B M_{-1,1,1,-2}$.

We have the following theorem, which was conjectured informally by Birman and Menasco:

Theorem 2.8 There are infinitely many $(x, y, z, w)$ 's such that the MFW-inequality is not sharp on $B M_{x, y, z, w}$.

Lemma 2.9 We have $D_{B M_{x, y, z, w}}^{+} \geq 2$.

Proof of Lemma 2.9 Change the BM diagram into the diagram in sketch (1) of Figure 5 by braid isotopy and denote it by $K$. Focus on the crossing shaded in sketch (1). Regard $K=K_{-}$. We can apply positive destabilization once to $K_{+}$and obtain the diagram in sketch (2-2). We also can apply positive destabilization once to $K_{0}$ as we can see in the passage sketch (3-1) $\Rightarrow(3-2) \Rightarrow$ (3-3). Therefore by Theorem 2.2 we have $D_{B M_{x, y, z, w}}^{+} \geq 2$ for any $(x, y, z, w)$. 


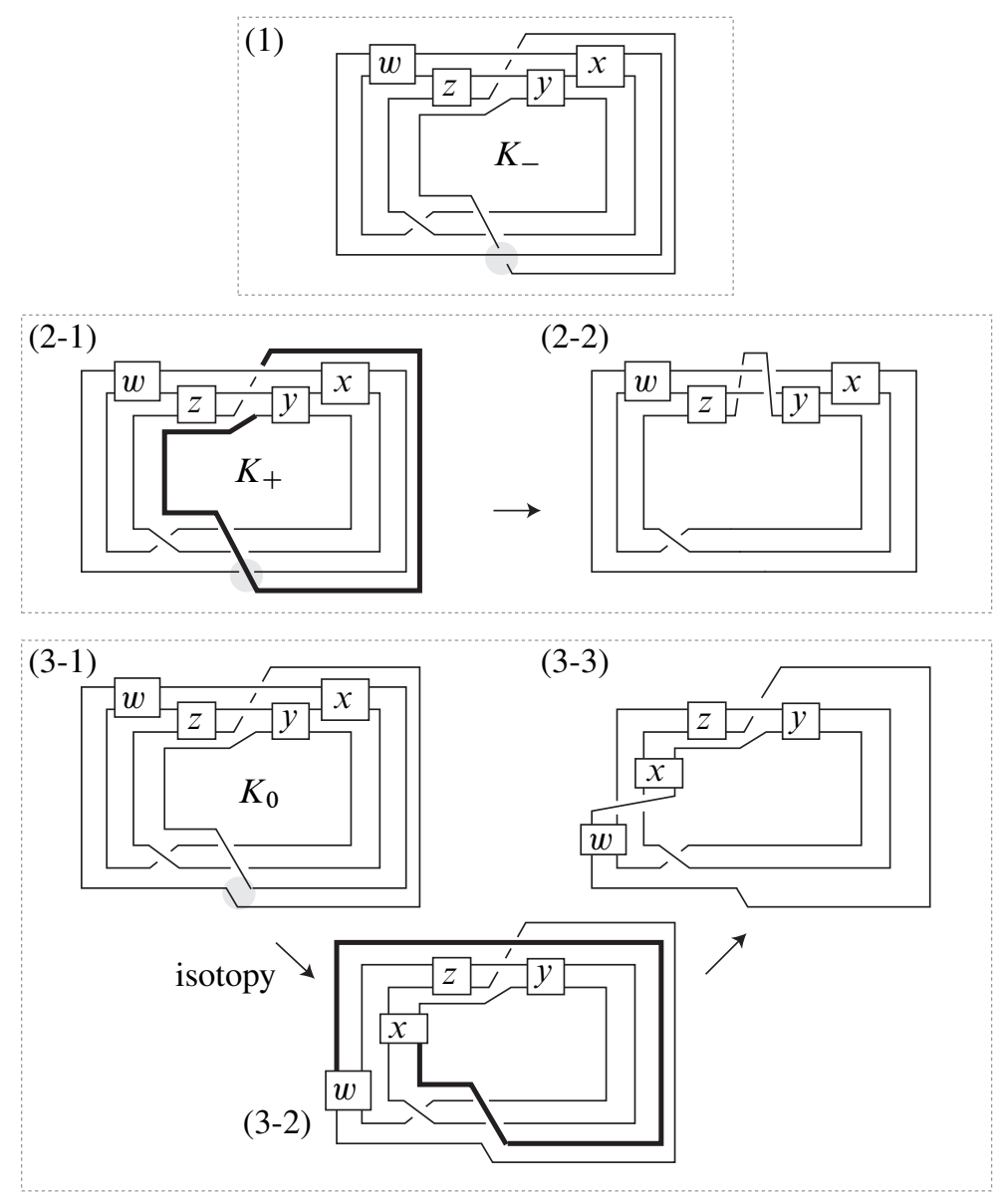

Figure 5: The BM-diagram satisfies the sufficient condition

It remains to prove that there exist infinitely many $(x, y, z, w)$ 's such that the braid index of $B M_{x, y, z, w}$ is 4 . We introduce $\mathcal{K}_{n}:=B M_{-1,-2, n, 2}$ and will show that for all $m \geq 1$ the braid index of $\mathcal{K}_{2 m}$ is 4 . (Note that $\mathcal{K}_{-2}=9_{42}, \mathcal{K}_{2}=10_{150}$ and $\mathcal{K}_{2 m}$ is a knot.) It will then follow, thanks to Lemma 2.9, that the MFW-inequality cannot be sharp on any $\mathcal{K}_{2 m}, m \geq 1$.

In order to do this, we use the enhanced Milnor number $\lambda$ defined by Neumann and Rudolph [16]. Recall that the fiber surface of a fiber knot is obtained by plumbing and deplumbing Hopf bands (see Giroux [7]). This $\lambda$ is an invariant of fibered knots and links counting algebraically the number of negative Hopf bands to get the fiber surface. 
(1)

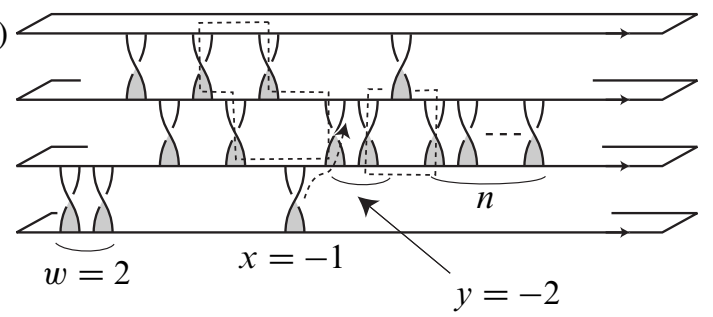

(2)

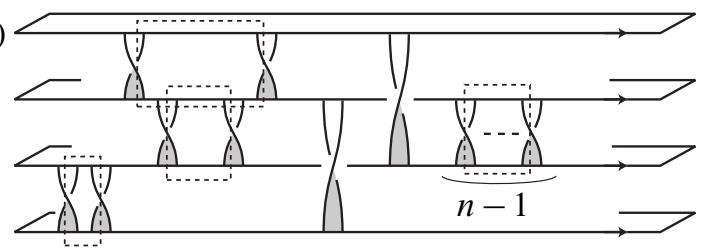

(3)

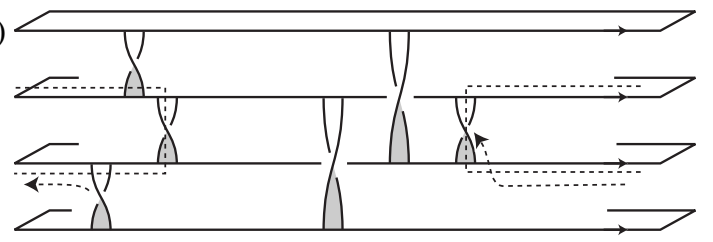

(4)

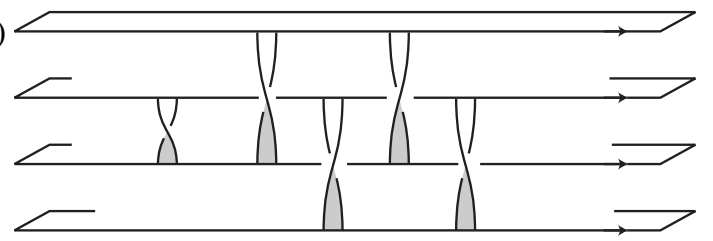

(5)

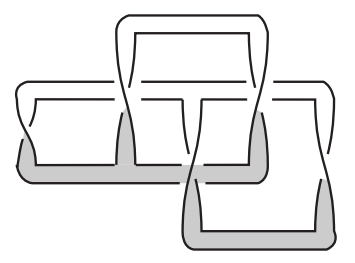

(10)

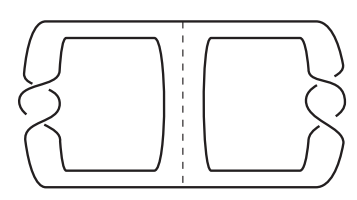

(9)

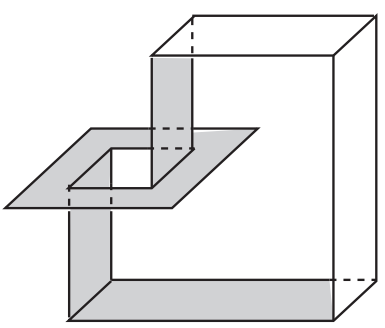

(8)

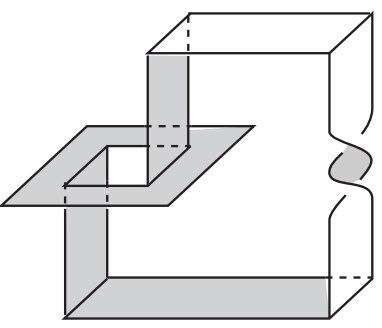

(7)

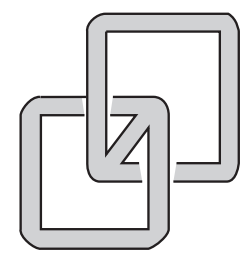

(6)

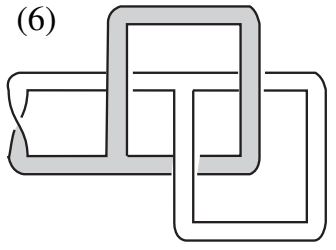

Figure 6: Deformation of $\mathcal{K}_{n}$ 
Lemma 2.10 All $_{\mathcal{K}_{n}}(n \geq 2)$ are fibered and have enhanced Milnor number $\lambda=1$.

Proof of Lemma 2.10 Sketch (1) of Figure 6 is the standard Bennequin surface of $\mathcal{K}_{n}$. We compress it twice as in the passage sketch (1) $\Rightarrow(2)$ along the disks bounded by dotted circles in sketch (1). Next, deplumb positive Hopf bands as much as possible as in the passage sketch (2) $\Rightarrow(3) \Rightarrow(4)=(5)$. Then isotope the surface until we get sketch (8). These operations do not change the enhanced Milnor number.

We apply Melvin and Morton's trick [11] p.167, as in the passage sketch (8) $\Rightarrow(9)$. We remark that the enhanced Milnor number is invariant under this trick.

The surface of sketch $(9)=(10)$, whose boundary is Pretzel link $P(-2,0,2)$, is plumbing of a positive Hopf band and a negative Hopf band. Thus it has $\lambda=1$ so does $\mathcal{K}_{n}$.

Here we summarize Xu's classification of 3-braids [21]. Let $\sigma_{1}, \sigma_{2}$ be the standard generators of $B_{3}$ the braid group of 3-strings satisfying $\sigma_{1} \sigma_{2} \sigma_{1}=\sigma_{2} \sigma_{1} \sigma_{2}$. Let $a_{1}:=\sigma_{1}, a_{2}:=\sigma_{2}$ and $a_{3}:=\sigma_{2} \sigma_{1} \sigma_{2}^{-1}$. We can identify them with the twisted bands in Figure 7.

Let $\alpha:=a_{1} a_{3}=a_{2} a_{1}=a_{3} a_{2}$. If $w \in B_{3}$ let $\bar{w}$ denote $w^{-1}$.

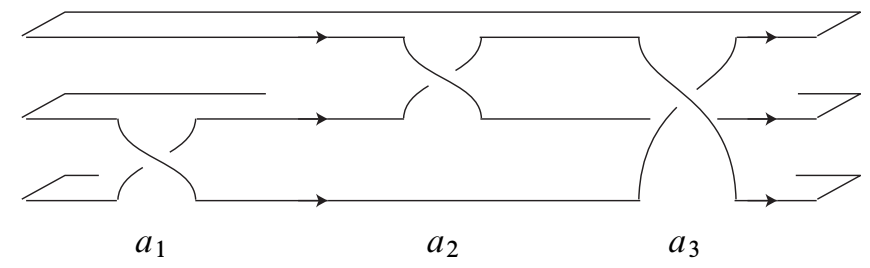

Figure 7: Xu's band generators

Theorem 2.11 (Xu [21]) Every conjugacy class in $B_{3}$ can be represented by a shortest word in $a_{1}, a_{2}, a_{3}$ uniquely up to symmetry. And the word has one of the three forms:

$$
\text { (1) } \alpha^{k} P, \quad \text { (2) } N \bar{\alpha}^{k}, \quad \text { (3) } N P \text {. }
$$

where $k \geq 0$ and $\bar{N}, P$ are positive words and the arrays of subscripts of the words are non-decreasing.

The next is another lemma for Theorem 2.8: 
Lemma 2.12 If a closed 3-braid has $\lambda=1$ and is a knot, then up to symmetry it has one of the following forms:

$$
\begin{aligned}
A_{x} & :=\overline{a_{3}} \overline{a_{2}}\left(a_{1}\right)^{x}, \quad x \geq 2, \text { even, } \\
B_{x, y} & :=\overline{a_{3}} \overline{a_{3}}\left(a_{1}\right)^{x}\left(a_{2}\right)^{y}, \quad x, y \geq 3, \text { odd, } \\
C_{x, y, z} & :=\overline{a_{2}}\left(a_{1}\right)^{x}\left(a_{2}\right)^{y}\left(a_{3}\right)^{z}, \quad x+z=\text { odd, } y=\text { even, } x, y, z \geq 1, \\
D_{x, y, z, w} & :=\overline{a_{2}}\left(a_{1}\right)^{x}\left(a_{2}\right)^{y}\left(a_{3}\right)^{z}\left(a_{1}\right)^{w}, \quad x, y \geq 2, z, w \geq 1 .
\end{aligned}
$$

Proof of Lemma 2.12 Assume we have a word $w \in B_{3}$. By Theorem 2.11, $w$ has one of the following forms:

\begin{tabular}{|l|ll|}
\hline Case (1)-1 & $w=\alpha^{k}$ & $k \geq 1$ \\
\hline Case (1)-2 & $w=\alpha^{k} P$ & $k \geq 1$ \\
\hline Case (1)-3 & $w=P$ & no $\alpha$ part \\
\hline Case (2)-1 & $w=\bar{\alpha}^{k}$ & $k \geq 1$ \\
\hline Case (2)-2 & $w=N \bar{\alpha}^{k}$ & $k \geq 1$ \\
\hline Case (2)-2 & $w=N$ & no $\bar{\alpha}$ part \\
\hline Case (3) & $w=N P$ & 18 cases to study \\
\hline
\end{tabular}

In this proof, we use the simplified notations:

\begin{tabular}{|c|l|c|}
\hline symbol & meaning & change in $\lambda$ \\
\hline$i$ & $a_{i}$ for $i=1,2,3$. & - \\
\hline$=$ & same conjugacy class & 0 \\
\hline$\longrightarrow$ & deplumb positive-Hopf bands & 0 \\
\hline$\Longrightarrow$ & deplumb negative-Hopf bands & $\geq 1$ \\
\hline$\approx$ & Melvin-Morton trick [11] & 0 \\
\hline$\rightsquigarrow$ & composition of deplumbings of \pm Hopf bands & $\geq 0$ \\
\hline
\end{tabular}

These are formulae we use:

$$
\begin{aligned}
& \alpha^{2} \longrightarrow \alpha, \text { Figure } 8 \\
& \alpha 123 \longrightarrow \alpha, \text { Figure } 9 \\
& \bar{i}(i-1) i \approx \bar{i} \overline{i-1} i, \text { Melvin-Morton trick } \\
& i(i+1) \bar{i} \approx i \overline{i+1} \bar{i}, \text { Melvin-Morton trick }
\end{aligned}
$$




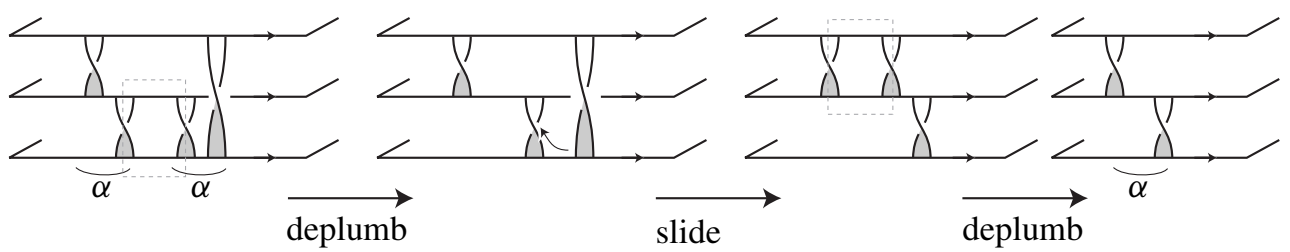

Figure 8: $\alpha^{2} \longrightarrow \alpha$

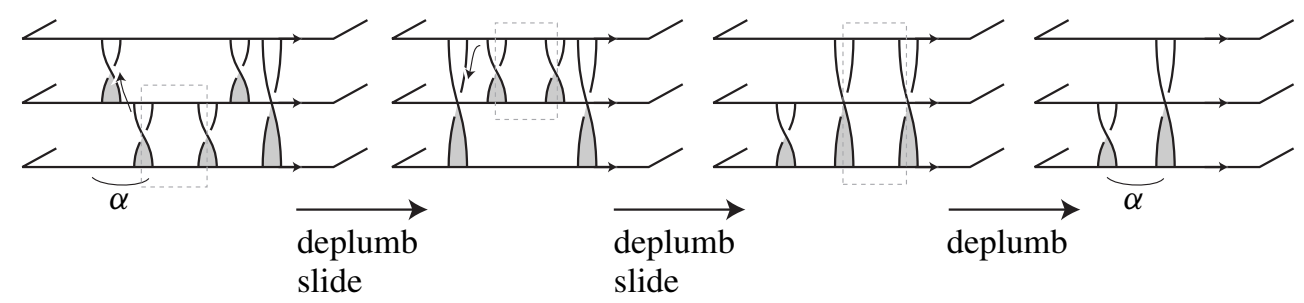

Figure 9: $\alpha 123 \longrightarrow \alpha$

Now we study each case.

Case (1)-1 By (13), we have $w=\alpha^{k} \longrightarrow \alpha$ (= unknot). Thus $w$ has $\lambda=0$.

Case (1)-2 By (13) up to permutation of $\{1,2,3\}$ we have $\alpha^{k} P \longrightarrow \alpha P \longrightarrow$ $\alpha(123123 \cdots \cdots)$. Thanks to (14) we have

$$
\alpha \overbrace{123123 \cdots \cdots}^{\text {length }=l} \rightarrow \alpha \overbrace{123123 \cdots \cdots}^{\text {length }=l-3} \text { for } l \geq 3 .
$$

If $l=1,2$, we have $\alpha 1=211 \longrightarrow \alpha$ and $\alpha 12=2112 \longrightarrow \alpha$. Thus $w$ has $\lambda=0$.

Case (1)-3 Assume $w=P$. There are three possible cases:

$$
P \longrightarrow(123)^{n}, \quad P \longrightarrow(123)^{n} 1 \quad \text { and } \quad P \longrightarrow(123)^{n} 12 \quad \text { where } n \geq 0 .
$$

If $w$ satisfies the first case, it is proved that $(123)^{n}$ is not fibered in Theorem 3.2 of [18], where Stoimenow determines fibreness of strongly quasi-positive 3-braid links. Therefore, $w$ is not fibered.

The second case can be reduced to the first case, since $(123)^{n} 1=1(123)^{n} \longrightarrow(123)^{n}$. For the third case, since $(123)^{n} 12=2(123)^{n} 1=\alpha(231)^{n} \longrightarrow \alpha, w$ has $\lambda=0$.

Case (2)-1 By (13), $w=\bar{\alpha}^{k} \Longrightarrow \bar{\alpha}$ and $w$ has $\lambda=2(k-1) \neq 1$. 
Case (2)-2 Suppose $w=N \bar{\alpha}^{k}$ where $k \geq 1$.

If $w=\bar{i} \bar{\alpha}$, we have $\bar{i} \bar{\alpha} \Longrightarrow \bar{\alpha}$ and $w$ has $\lambda=1$. However, the closure of $w$ has more than one component and it does not satisfy the condition of the lemma.

If $w \neq \bar{i} \bar{\alpha}$, we have $N \bar{\alpha}^{k} \Longrightarrow \bar{\alpha}$ by (14), and $w$ has $\lambda \geq 2$.

Case (2)-3 Suppose $w=N$. There are three possible cases:

$$
N \Longrightarrow(\overline{3} \overline{2} \overline{1})^{n}, \quad N \Longrightarrow(\overline{3} \overline{2} \overline{1})^{n} \overline{3} \quad \text { and } \quad N \Longrightarrow(\overline{3} \overline{2} \overline{1})^{n} \overline{3} \overline{2} \quad \text { where } n \geq 0 \text {. }
$$

For the first case, $w$ is not fibered [18].

For the second case, if $n=0$ then $w$ has $\lambda=1$ if and only if $w=\overline{3} \overline{3}$. However this has two components. If $n \geq 1$, since $(\overline{3} \overline{2} \overline{1})^{n} \overline{3} \Longrightarrow(\overline{3} \overline{2} \overline{1})^{n}$ it can be reduced to the first case.

For the third case, if $n=0$ then $w$ has $\lambda=1$ if and only if $w=\overline{3} \overline{3} \overline{2}$. However it has two components. If $n \geq 1$, we have $(\overline{3} \overline{2} \overline{1})^{n} \overline{3} \overline{2}=\overline{2} \overline{3}(\overline{2} \overline{1} \overline{3})^{n}=\bar{\alpha}(\overline{2} \overline{1} \overline{3})^{n} \Longrightarrow \bar{\alpha}$ and $w$ has $\lambda \geq 3 n$.

Case (3) Assume $w=N P$. Let $w^{\prime}$ be a word obtained from $w$ by deplumbing \pm Hopf bands sufficiently enough times, ie, $w \rightsquigarrow w^{\prime}$. This $w^{\prime}$ has one of the following 18 forms up to permutation of $\{1,2,3\}$.

\begin{tabular}{|l|ll|}
\hline case & $w^{\prime}$ & \\
\hline i & $(\overline{2} \overline{1} \overline{3})^{k}(123)^{l}$ & $k, l \geq 1$ \\
\hline ii & $(\overline{2} \overline{1} \overline{3})^{k}(123)^{l} 1$ & $k \geq 1, l \geq 0$ \\
\hline iii & $(\overline{2} \overline{1} \overline{3})^{k}(123)^{l} 12$ & $k \geq 1, l \geq 0 \quad$ not shortest word \\
\hline iv & $\overline{3}(\overline{2} \overline{1} \overline{3})^{k}(123)^{l}$ & $k \geq 0, l \geq 1 \quad$ not shortest word \\
\hline v & $\overline{3}(\overline{2} \overline{1} \overline{3})^{k}(123)^{l} 1$ & $k, l \geq 0$ \\
\hline vi & $\overline{3}(\overline{2} \overline{1} \overline{3})^{k}(123)^{l} 12$ & $k, l \geq 0$ \\
\hline vii & $\overline{1} \overline{3}(\overline{2} \overline{1} \overline{3})^{k}(123)^{l}$ & $k \geq 0, l \geq 1$ \\
\hline viii & $\overline{1} \overline{3}(\overline{2} \overline{1} \overline{3})^{k}(123)^{l} 1$ & $k, l \geq 0$ \\
\hline ix & $\overline{1} \overline{3}(\overline{2} \overline{1} \overline{3})^{k}(123)^{l} 12$ & $k, l \geq 0$ \\
\hline
\end{tabular}

Algebraic $8 \mathcal{G}$ Geometric Topology, Volume 6 (2006) 


\begin{tabular}{|l|ll|}
\hline i' $^{\prime}$ & $(\overline{1} \overline{3} \overline{2})^{k}(123)^{l}$ & $k, l \geq 1$ \\
\hline ii $^{\prime}$ & $(\overline{1} \overline{3} \overline{2})^{k}(123)^{l} 1$ & $k \geq 1, l \geq 0 \quad$ not shortest word \\
\hline iii' $^{\prime}$ & $(\overline{1} \overline{3} \overline{2})^{k}(123)^{l} 12$ & $k \geq 1, l \geq 0$ \\
\hline iv $^{\prime}$ & $\overline{2}(\overline{1} \overline{3} \overline{2})^{k}(123)^{l}$ & $k \geq 0, l \geq 1$ \\
\hline $\mathrm{v}^{\prime}$ & $\overline{2}(\overline{1} \overline{3} \overline{2})^{k}(123)^{l} 1$ & $k, l \geq 0$ \\
\hline vi' $^{\prime}$ & $\overline{2}(\overline{1} \overline{3} \overline{2})^{k}(123)^{l} 12$ & $k, l \geq 0$ \\
\hline vii $^{\prime}$ & $\overline{3} \overline{2}(\overline{1} \overline{3} \overline{2})^{k}(123)^{l}$ & $k \geq 0, l \geq 1 \quad$ not shortest word \\
\hline viii' $^{\prime}$ & $\overline{3} \overline{2}(\overline{1} \overline{3} \overline{2})^{k}(123)^{l} 1$ & $k, l \geq 0$ \\
\hline ix $^{\prime}$ & $\overline{3} \overline{2}(\overline{1} \overline{3} \overline{2})^{k}(123)^{l} 12$ & $k, l \geq 0$ \\
\hline
\end{tabular}

Since words of case iii, iv, viii, $\mathrm{ii}^{\prime}$, $\mathrm{vi}^{\prime}$ and $\mathrm{vii}^{\prime}$ are not shortest (reducible) we eliminate them from the list.

These are reduction formulae we use:

$$
\begin{aligned}
& (\overline{2} \overline{1} \overline{3})(123) \stackrel{(22)}{\longrightarrow} \overline{2}(3 \overline{2})=1 \overline{2} \overline{2} \Longrightarrow 1 \overline{2} \\
& (1 \overline{2})(123) \stackrel{(31)}{\longrightarrow} 11 \overline{2} \longrightarrow 1 \overline{2} \\
& (\overline{2} \overline{1} \overline{3})(1 \overline{2}) \stackrel{(20)}{\longrightarrow} 1 \overline{3} \overline{2}=\overline{2} \overline{2} 3 \Longrightarrow=1 \overline{2} \\
& (\overline{2} \overline{1} \overline{3}) 1 \approx \overline{2} \overline{1} 31=1 \overline{2} \overline{2} 1 \Longrightarrow=11 \overline{3} \longrightarrow 1 \overline{3} \\
& (\overline{2} \overline{1} \overline{3}) 1 \overline{3} \stackrel{(20)}{\longrightarrow} 1 \overline{3} \overline{3} \Longrightarrow 1 \overline{3} \\
& \overline{1} \overline{3}(123)=\overline{1} 22 \overline{1} 3 \longrightarrow \overline{1} 2 \overline{1} 3=3 \overline{1} \overline{1} 3 \Longrightarrow=33 \overline{2} \longrightarrow 3 \overline{2} \\
& 3 \overline{2}(123) \stackrel{(31)}{\longrightarrow} 3(1 \overline{2})=\overline{1} 33 \longrightarrow \overline{1} 3=3 \overline{2} \\
& (123) 1 \overline{3} \approx 123 \overline{1} \overline{3}=1 \overline{3} 22 \overline{3} \\
& \longrightarrow 1 \overline{3} 2 \overline{3}=1 \overline{3} \overline{3} 1 \Longrightarrow 1 \overline{3} 1 \longrightarrow 1 \overline{3} \\
& 12 \overline{1} \overline{3}=1 \overline{3} \overline{3} 1 \Longrightarrow 1 \overline{3} 1 \longrightarrow \overline{2} 1=1 \overline{3} \\
& (\overline{1} \overline{3} \overline{2})(123) \stackrel{(29)}{\longrightarrow} 3 \overline{1} 3=33 \overline{2} \Longrightarrow 3 \overline{2}=\overline{1} 3 \\
& (\overline{1} \overline{3} \overline{2}) \overline{1} 3=\overline{1} \overline{3} 1 \overline{2} \overline{2} \Longrightarrow \approx \overline{1} 31 \overline{2}=\overline{1} \overline{1} 33 \longrightarrow \Longrightarrow \overline{1} 3 \\
& \overline{1} 3(123) \approx \overline{1} \overline{3} 123=\overline{1} 22 \overline{1} 3 \longrightarrow 3 \overline{1} 3 \longrightarrow 3 \overline{2}=\overline{1} 3 \\
& (\overline{1} \overline{3} \overline{2}) 12=\overline{1} 2 \overline{3} \overline{3} 2 \Longrightarrow=\overline{1} 22 \overline{1} \longrightarrow \overline{1} 2 \overline{1}=3 \overline{1} \overline{1} \Longrightarrow 3 \overline{1} \\
& (\overline{1} \overline{3} \overline{2}) 3 \overline{1} \stackrel{(19)}{\longrightarrow} 3 \overline{1} \quad \text { permutation of (19) } \\
& \overline{2}(123) \approx \overline{2} \overline{1} 23=\overline{2} 33 \overline{2} \longrightarrow \overline{2} 3 \overline{2}=1 \overline{2} \overline{2} \Longrightarrow 1 \overline{2} \\
& 1 \overline{2}(\overline{1} \overline{3} \overline{2}) \approx 12 \overline{1} \overline{3} \overline{2}=1 \overline{3} \overline{3} 1 \overline{2} \Longrightarrow=\overline{2} 11 \overline{2} \longrightarrow \overline{2} 1 \overline{2} \Longrightarrow 1 \overline{2}
\end{aligned}
$$

Algebraic $6 \mathcal{G}$ Geometric $\mathcal{T}$ opology, Volume 6 (2006) 
Sublemma 2.13 For $k, l \geq 1$, we have:

$$
\begin{aligned}
& (\overline{2} \overline{1} \overline{3})^{k}(123)^{l} \rightsquigarrow 1 \overline{2} \\
& (\overline{1} \overline{3} \overline{2})^{k}(123)^{l} \rightsquigarrow \overline{1} 3
\end{aligned}
$$

Either case, the increase of $\lambda$ is $\geq 2$.

Proof From (17), (19) and (20), we obtain (33). Similarly, (34) follows from (26), (27) and (28).

Case 3-i By (33), our $w$ is fibered and $\lambda \geq 2$.

Case 3-ii If $k \geq 1 . l=0$,

$$
w \rightsquigarrow w^{\prime}=(\overline{2} \overline{1} \overline{3})^{k} 1 \stackrel{(20)}{\longrightarrow}(\overline{2} \overline{1} \overline{3})^{k-1} 1 \overline{3} \stackrel{(21)}{\longrightarrow} \cdots \stackrel{(21)}{\longrightarrow} 1 \overline{3}
$$

and $w$ has $\lambda=1$ if and only if $w=\overline{2} \overline{1} \overline{3} 1^{x}$ for some $x \geq 1$. However it has 2 or 3 components and it does not satisfy the condition of Lemma 2.12. If $k, l \geq 1$,

$$
w \rightsquigarrow w^{\prime}=(\overline{2} \overline{1} \overline{3})^{k}(123)^{l} 1 \stackrel{(33)}{\longrightarrow}(1 \overline{2}) 1 \longrightarrow 1 \overline{2}
$$

and $w$ has $\lambda \geq 2$.

Case 3-v When $k=l=0, w$ has $\lambda=1$ if and only if $w=\overline{3} \overline{3} 1^{x}$ for some $x \geq 1$, which has more than 1 component. If $k \geq 1$ and $l=0$,

$$
w \rightsquigarrow w^{\prime}=\overline{3}(\overline{2} \overline{1} \overline{3})^{k} 1 \stackrel{(21)}{\longrightarrow} \cdots \stackrel{(21)}{\longrightarrow} 1 \overline{3}
$$

and $\lambda \geq 2$. If $k=0, l \geq 1$,

$$
w \rightsquigarrow w^{\prime}=\overline{3}(123)^{l} 1=(123)^{l} 1 \overline{3} \stackrel{(24)}{\longrightarrow} \cdots \stackrel{(24)}{\longrightarrow} 1 \overline{3} .
$$

Thus $w$ has $\lambda=1$ if and only if $w=\overline{3} 1^{x} 2^{y} 3^{z} 1^{w}$ for $x, y, z, w \geq 1$. If $k, l \geq 1$,

$$
w \rightsquigarrow w^{\prime}=\overline{3}(\overline{2} \overline{1} \overline{3})^{k}(123)^{l} 1 \stackrel{(33)}{\longrightarrow} \overline{3}(1 \overline{2}) 1=\overline{3} 11 \overline{3} \longrightarrow \Longrightarrow 1 \overline{3}
$$

and $\lambda \geq 2$.

Case 3-vi If $k=l=0$,

$$
w \rightsquigarrow w^{\prime}=\overline{3} 12=22 \overline{1} \longrightarrow 2 \overline{1} .
$$

Therefore, $w$ has $\lambda=1$ if and only if $w=\overline{3} \overline{3} 1^{x} 2^{y}$ for $x, y \geq 1$. If $k=0, l \geq 1$

$$
w \rightsquigarrow w^{\prime}=\overline{3}(123)^{l} 12=(123)^{l} 12 \overline{3} \longrightarrow(123)^{l} 1 \overline{3} \stackrel{(24)}{\longrightarrow} \cdots \stackrel{(24)}{\longrightarrow} 1 \overline{3}
$$


and $w$ has $\lambda=1$ if and only if $w=\overline{3} 1^{x} 2^{y} 3^{z} 1^{w} 2^{v}$ for some $x, y, z, w, v \geq 1$. When $k \geq 1, l=0$,

$$
w \rightsquigarrow w^{\prime}=\overline{3}(\overline{2} \overline{1} \overline{3})^{k} 12 \longrightarrow(\overline{2} \overline{1} \overline{3})^{k} 1 \overline{3} \stackrel{(21)}{\longrightarrow} \ldots \stackrel{(21)}{\longrightarrow} 1 \overline{3}
$$

and $\lambda \geq 2$. If $k, l \geq 1$,

$$
w \rightsquigarrow w^{\prime} \stackrel{(33)}{\longrightarrow} \overline{3}(1 \overline{2}) 12=\overline{3} 11 \overline{3} 2 \longrightarrow \overline{3} 1 \overline{3} 2=2 \overline{3} \overline{3} 2 \longrightarrow \Longrightarrow 2 \overline{3}
$$

and $\lambda \geq 2$.

Case 3-vii If $k=0, l \geq 1$,

$$
w \rightsquigarrow w^{\prime}=\overline{1} \overline{3}(123)^{l} \stackrel{(22)}{\longrightarrow} 3 \overline{2}(123)^{l-1} \stackrel{(23)}{\longrightarrow} \ldots \stackrel{(22)}{\longrightarrow} 3 \overline{2}
$$

and $\lambda=1$ if and only if $w=\overline{1} \overline{3} 1^{x} 2^{y} 3^{z}$ for some $x, y, z \geq 1$. If $k, l \geq 1$,

$$
w \rightsquigarrow w^{\prime} \stackrel{(33)}{\longrightarrow} \overline{1} \overline{3}(1 \overline{2})=\overline{2} \overline{1} \overline{3} 1 \stackrel{(20)}{\longrightarrow} 1 \overline{3}
$$

and $\lambda \geq 2$.

Case 3-ix When $k=l=0$,

$$
w \rightsquigarrow w^{\prime}=\overline{1} \overline{3} 12=\overline{1} 22 \overline{1} \longrightarrow \Longrightarrow \overline{1} 2 .
$$

Thus $w$ has $\lambda=1$ if and only if $w=\overline{1} \overline{3} 1^{x} 2^{y}$ for some $x, y \geq 1$. When $k=0, l \geq 1$

$$
w \rightsquigarrow w^{\prime}=\overline{1} \overline{3}(123)^{l} 12 \stackrel{(22)}{\longrightarrow} \stackrel{(23)}{\longrightarrow} \overline{1} 312=3 \overline{2} 12 \approx 3 \overline{2} \overline{1} 2=\overline{1} 33 \overline{1} \longrightarrow \Longrightarrow \overline{1} 3
$$

and $\lambda \geq 2$. When $k \geq 1, l=0$

$$
w \rightsquigarrow w^{\prime}=\overline{1} \overline{3}(\overline{2} \overline{1} \overline{3})^{k} 12=12 \overline{1} \overline{3}(\overline{2} \overline{1} \overline{3})^{k} \stackrel{(25)}{\longrightarrow} 1 \overline{3}(\overline{2} \overline{1} \overline{3})^{k} \stackrel{(21)}{\longrightarrow} \cdots \stackrel{(21)}{\longrightarrow} 1 \overline{3}
$$

and $\lambda \geq 2$. When $k, l \geq 1$

$$
w \rightsquigarrow w^{\prime} \stackrel{(33)}{\longrightarrow} \overline{1} \overline{3}(1 \overline{2}) 12=12 \overline{1} \overline{3} 1 \overline{2} \stackrel{(25)}{\longrightarrow} 1 \overline{3} 1 \overline{2}=\overline{2} 11 \overline{2} \longrightarrow \Longrightarrow 1 \overline{2}
$$

and $\lambda \geq 2$.

Case 3-i' By (34) our $w$ is fibered and $\lambda \geq 2$.

Case 3-iii' When $k>l=0$,

$$
w \rightsquigarrow w^{\prime}=(\overline{1} \overline{3} \overline{2})^{k} 12 \stackrel{(29)}{\longrightarrow}(\overline{1} \overline{3} \overline{2})^{k-1} 2 \overline{1} \stackrel{(30)}{\longrightarrow} \cdots \stackrel{(30)}{\longrightarrow} 3 \overline{1}
$$

and $\lambda \geq 2$. When $k, l \geq 1$,

$$
w \rightsquigarrow w^{\prime} \stackrel{(34)}{\longrightarrow}(\overline{1} 3) 12 \approx \overline{1} \overline{3} 12=\overline{1} 22 \overline{1} \longrightarrow \Longrightarrow 3 \overline{1}
$$


and $\lambda \geq 2$.

Case 3-iv' When $k=0, l \geq 1$,

$$
w \rightsquigarrow w^{\prime}=\overline{2}(123)^{l} \stackrel{(31)}{\longrightarrow} 1 \overline{2}(123)^{l-1} \stackrel{(18)}{\longrightarrow} \cdots \stackrel{(18)}{\longrightarrow} 1 \overline{2} .
$$

Thus $w$ has $\lambda=1$ if and only if $w=\overline{2} 1^{x} 2^{y} 3^{z}$ for $x, y, z \geq 1$. To make the braid closure one component, we further require $x+z=$ odd. If $k, l \geq 1$,

$$
w \rightsquigarrow w^{\prime} \stackrel{(34)}{\longrightarrow} \overline{2}(\overline{1} 3)=1 \overline{2} \overline{2} \Longrightarrow 1 \overline{2}
$$

and $\lambda \geq 2$.

Case 3-v' When $k=l=0$,

$$
w \rightsquigarrow w^{\prime}=\overline{2} 1 .
$$

Thus $w$ has $\lambda=1$ if and only if $w=\overline{2} \overline{2} 1^{x}$, which has 2 or 3 components. When $k=0$ and $l \geq 1$,

$$
w \rightsquigarrow w^{\prime}=\overline{2}(123)^{l} 1 \stackrel{(31)}{\longrightarrow} 1 \overline{2}(123)^{l-1} 1 \stackrel{(18)}{\longrightarrow} \cdots \stackrel{(18)}{\longrightarrow} 1 \overline{2} 1=11 \overline{2} \longrightarrow 1 \overline{2}
$$

thus $w$ has $\lambda=1$ if and only if $w=\overline{2} 1^{x} 2^{y} 3^{z} 1^{w}$ for some $x, y, z, w \geq 1$. When $k \geq 1, l=0$,

$$
w \rightsquigarrow w^{\prime}=\overline{2}(\overline{1} \overline{3} \overline{2})^{k} 1=1 \overline{2}(\overline{1} \overline{3} \overline{2})^{k} \stackrel{(32)}{\longrightarrow} \cdots \stackrel{(32)}{\longrightarrow} 1 \overline{2}
$$

and $\lambda \geq 2$. When $k, l \geq 1$

$$
w \rightsquigarrow w^{\prime} \stackrel{(34)}{\longrightarrow} \overline{2}(\overline{1} 3) 1 \Longrightarrow 1 \overline{2} 1 \longrightarrow 1 \overline{2}
$$

and $\lambda \geq 2$.

Case 3-viii' When $k=l=0$,

$$
w \rightsquigarrow w^{\prime}=\overline{3} \overline{2} 1=1 \overline{3} \overline{2}=\overline{2} 1 \overline{2} \Longrightarrow 1 \overline{2}
$$

Thus $w$ has $\lambda=1$ if and only if $w=\overline{3} \overline{2} 1^{x}$ for some $x \geq 1$. When $k=0$ and $l \geq 1$,

$$
w \rightsquigarrow w^{\prime}=\overline{3} \overline{2}(123)^{l} 1=1 \overline{3} \overline{2}(123)^{l} \Longrightarrow 1 \overline{2}(123)^{l} \stackrel{(18)}{\longrightarrow} \cdots \stackrel{(18)}{\longrightarrow} 1 \overline{2}
$$

and $\lambda \geq 2$. When $k \geq 1$ and $l=0$,

$$
w \rightsquigarrow w^{\prime}=\overline{3} \overline{2}(\overline{1} \overline{3} \overline{2})^{k} 1 \Longrightarrow 1 \overline{2}(\overline{1} \overline{3} \overline{2})^{k} \stackrel{(32)}{\longrightarrow} \cdots \stackrel{(32)}{\longrightarrow} 1 \overline{2}
$$

and $\lambda \geq 2$. When $k, l \geq 1$

$$
w \rightsquigarrow w^{\prime} \stackrel{(34)}{\longrightarrow} \overline{3} \overline{2}(\overline{1} 3) 1 \Longrightarrow 1 \overline{2} \overline{1} 3=\overline{2} 33 \overline{2} \Longrightarrow \longrightarrow \overline{2} 3=1 \overline{2} .
$$


and $\lambda \geq 2$.

Case 3-ix' $\quad$ When $k=l=0$,

$$
w \rightsquigarrow w^{\prime}=\overline{3} \overline{2} 12=2 \overline{3} \overline{3} 2 \Longrightarrow \longrightarrow 2 \overline{3} \text {. }
$$

Thus $w$ has $\lambda=1$ if and only if $w=\overline{3} \overline{2} 1^{x} 2^{y}$ for some $x, y \geq 1$. When $k=0, l \geq 1$,

$$
\begin{aligned}
w & \rightsquigarrow w^{\prime}=\overline{3} \overline{2}(123)^{l} 12=12 \overline{3} \overline{2}(123)^{l}=\overline{2} 11 \overline{2}(123)^{l} \longrightarrow \Longrightarrow \overline{2} 3(123)^{l} \\
& =1 \overline{2}(123)^{l} \stackrel{(18)}{\longrightarrow} \cdots \stackrel{(18)}{\longrightarrow} 1 \overline{2}
\end{aligned}
$$

and $\lambda \geq 2$. When $k \geq 1, l=0$,

$$
w \rightsquigarrow w^{\prime}=\overline{3} \overline{2}(\overline{1} \overline{3} \overline{2})^{k} 12 \longrightarrow \Longrightarrow 1 \overline{2}(\overline{1} \overline{3} \overline{2})^{k} \stackrel{(32)}{\longrightarrow} \cdots \stackrel{(32)}{\longrightarrow} 1 \overline{2}
$$

and $\lambda \geq 2$. When $k, l \geq 1$

$$
w \rightsquigarrow w^{\prime} \stackrel{(34)}{\longrightarrow} \overline{3} \overline{2}(\overline{1} 3) 12 \longrightarrow \Longrightarrow 1 \overline{2}(\overline{1} 3)=11 \overline{2} \overline{2} \longrightarrow \Longrightarrow 1 \overline{2}
$$

and $\lambda \geq 2$.

Table 1 summarizes all the words with $\lambda=1$.

Words $A_{x}, \cdots, D_{x, y, z, w}$ are defined in Table 1 . We can see that any word with $\lambda=1$ and having one component has one of the forms; $A_{x}, \cdots, D_{x, y, z, w}$.

Lemma 2.14 The leading terms of the Alexander polynomials of $\mathcal{K}_{n}, A_{x}, B_{x, y}$, $C_{x, y, z}$ and $D_{x, y, z, w}$ are the following:

$$
\begin{gathered}
\mathcal{K}_{n} ; \pm\left(1-4 t-6 t^{2}+8 t^{3}-\cdots\right) \quad \text { if } n \geq 2, \\
A_{x} ; \pm(1-3 t+\cdots) \quad \text { if } x \geq 2, \\
B_{x, y} ; \pm(1-3 t+\cdots) \quad \text { if } x, y \geq 3, \\
C_{x, y, z} ; \pm(1-5 t+\cdots) \quad \text { if } x, y, z \geq 2, \\
C_{1,2, z}, C_{1, y, 2}, C_{2, y, 1}, C_{x, 2,1} ; \pm\left(1-4 t+6 t^{2}-7 t^{3}+\cdots\right) \quad \text { if } x, y, z \geq 4, \\
C_{1, y, z}, C_{x, y, 1} ; \pm\left(1-4 t+7 t^{2}+\cdots\right) \quad \text { if } x, y, z \geq 3 \\
D_{x, y, z, w}, D_{x, y, z, 1} ; \pm(1-6 t+\cdots) \quad \text { if } x, y, z, w \geq 2, \\
D_{x, y, 1, w} ; \pm(1-5 t+\cdots) \quad \text { if } x, y, w \geq 2 .
\end{gathered}
$$

In particular, $\mathcal{K}_{n} \neq A_{x}, B_{x, y}, C_{x, y, z}, D_{x, y, z, w}$. 
Table 1

\begin{tabular}{|c|c|}
\hline case & word with $\lambda=1$. \\
\hline $\mathrm{i}$ & none. \\
\hline ii & $\overline{2} \overline{1} \overline{3} 1^{x}$ (2 or 3 components.) \\
\hline $\mathrm{V}$ & $\begin{array}{l}\overline{3} 1^{x} 2^{y} 3^{z} 1^{w}= \begin{cases}C_{x+1, y, z} & \text { when } w=1 \\
D_{x+1, y, z, w-1} & \text { when } w \geq 2 .\end{cases} \\
\overline{3} \overline{3} 1^{x}(2 \text { or } 3 \text { components. })\end{array}$ \\
\hline vi & $\begin{array}{l}\overline{3} \overline{3} 1^{x} 2^{y}=: B_{x, y} . \\
\overline{3} 1^{x} 2^{y} 3^{z} 1^{w} 2^{v}= \begin{cases}C_{x+v+1, y, z} & \text { when } w=1, \\
D_{x+v+1, y, z, w-1} & \text { when } w \geq 2 .\end{cases} \end{array}$ \\
\hline vii & $\overline{1} \overline{3} 1^{x} 2^{y} 3^{z}=\overline{1} \overline{3} 1^{x+z} 2^{y}$ \\
\hline ix & $\overline{1} \overline{3} 1^{x} 2^{y}= \begin{cases}\overline{3} \overline{3} 1^{x+1} & \text { when } y=1 \quad(2 \text { or } 3 \text { components }) \\
B_{x+1, y-1} & \text { when } y \geq 2\end{cases}$ \\
\hline $\mathrm{i}^{\prime}$ & none. \\
\hline iii' $^{\prime}$ & none. \\
\hline iv $^{\prime}$ & $\overline{2} 1^{x} 2^{y} 3^{z}=: C_{x, y, z}$ \\
\hline $\mathrm{v}^{\prime}$ & $\begin{array}{l}\overline{2} 1^{x} 2^{y} 3^{z} 1^{w}=: D_{x, y, z, w} . \\
\overline{2} \overline{2} 1^{x}(2 \text { or } 3 \text { components. })\end{array}$ \\
\hline viii' $^{\prime}$ & $\overline{3} \overline{2} 1^{x}=: A_{x}$. \\
\hline $\mathrm{ix}^{\prime}$ & $\overline{3} \overline{2} 1^{x} 2^{y}= \begin{cases}\overline{3} \overline{3} 2^{y+1} & \text { when } x=1, \quad(2 \text { or } 3 \text { components }) \\
B_{x-1, y+1} & \text { when } x \geq 2 .\end{cases}$ \\
\hline
\end{tabular}

Proof of Lemma 2.14 We prove that the Alexander polynomial of $C_{x, y, z}$ for $x, y, z \geq$ 2 is $\pm(1-5 t+\cdots)$. Recall that Xu's Bennequin surface is a minimal genus Seifert surface. Let $F$ be the Bennequin surface of $C_{x, y, z}$ and choose a basis

$$
\left\{u^{(1)}, u^{(2)}, u_{1}^{(3)}, \cdots, u_{x-1}^{(3)}, u_{1}^{(4)}, \cdots, u_{y-1}^{(4)}, u_{1}^{(5)}, \cdots, u_{z-1}^{(5)}\right\}
$$

for $H_{1}(F)$ as in Figure 10, where $u^{(k)}(k=1, \cdots, 5)$ corresponds to loop $(k)$. 
With respect to the basis, let $V_{x, y, z}$ denote the Seifert matrix for $C_{x, y, z}$.

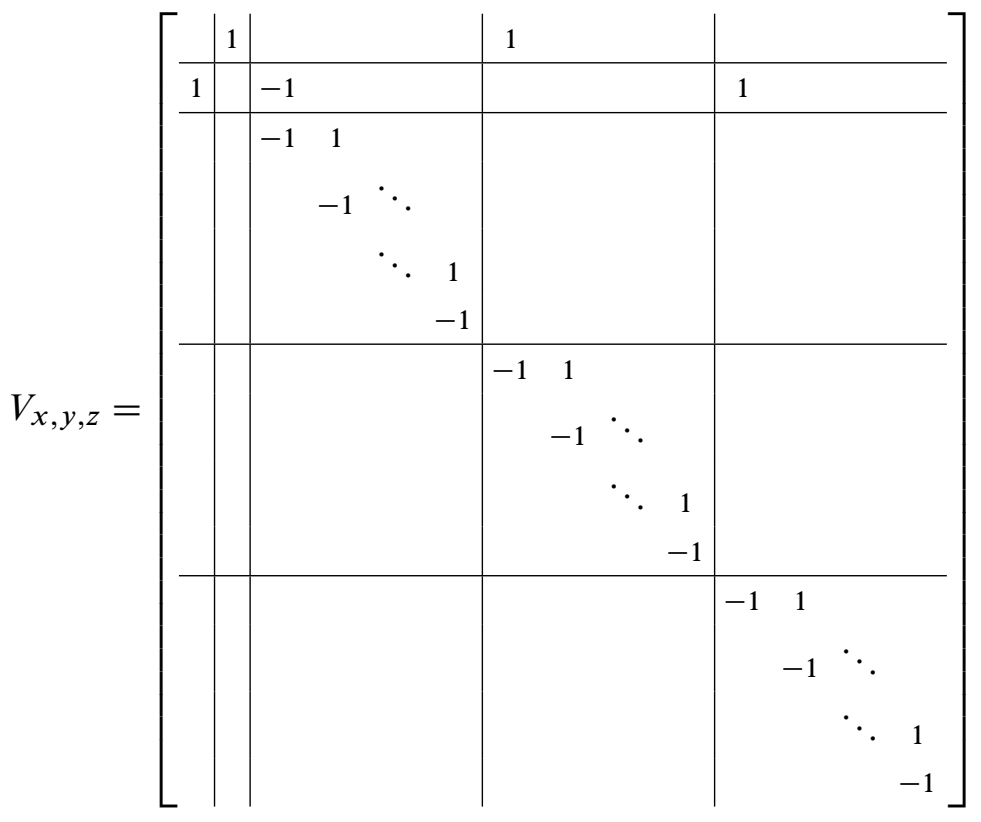

The empty spaces contain only 0 's. The 3 rd (resp. 4 th, 5 th) diagonal block has size $(x-1) \times(x-1)(\operatorname{resp} .(y-1) \times(y-1),(z-1) \times(z-1))$. The Alexander polynomial satisfies:

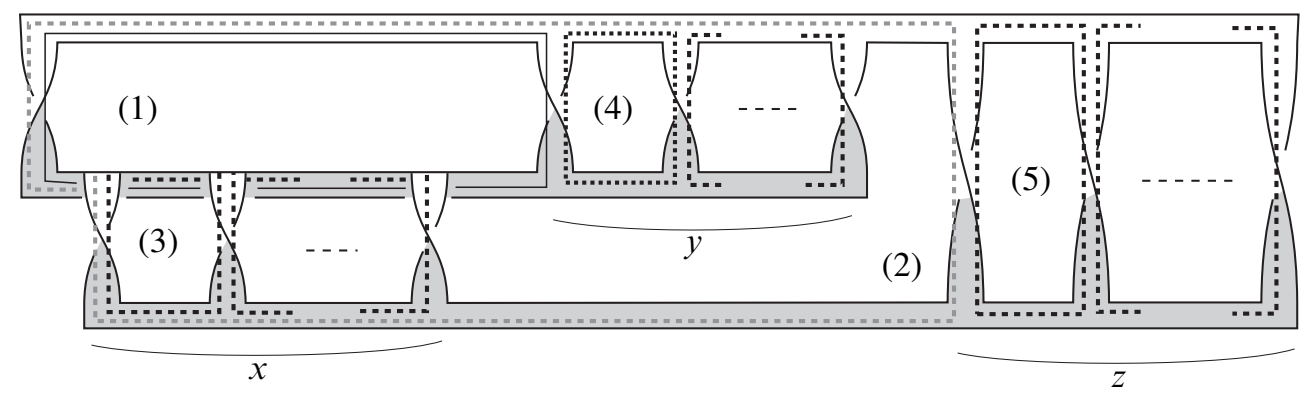

Figure 10: The Bennequin surface $F$ of $C_{x, y, z}=\overline{2} 1^{x} 2^{y} 3^{z}$ and a basis for $H_{1}(F)$

Algebraic 83 Geometric Topology, Volume 6 (2006) 
$\Delta_{x, y, z}(t)=\operatorname{det}\left(V_{x, y, z}^{T}-t V_{x, y, z}\right)$

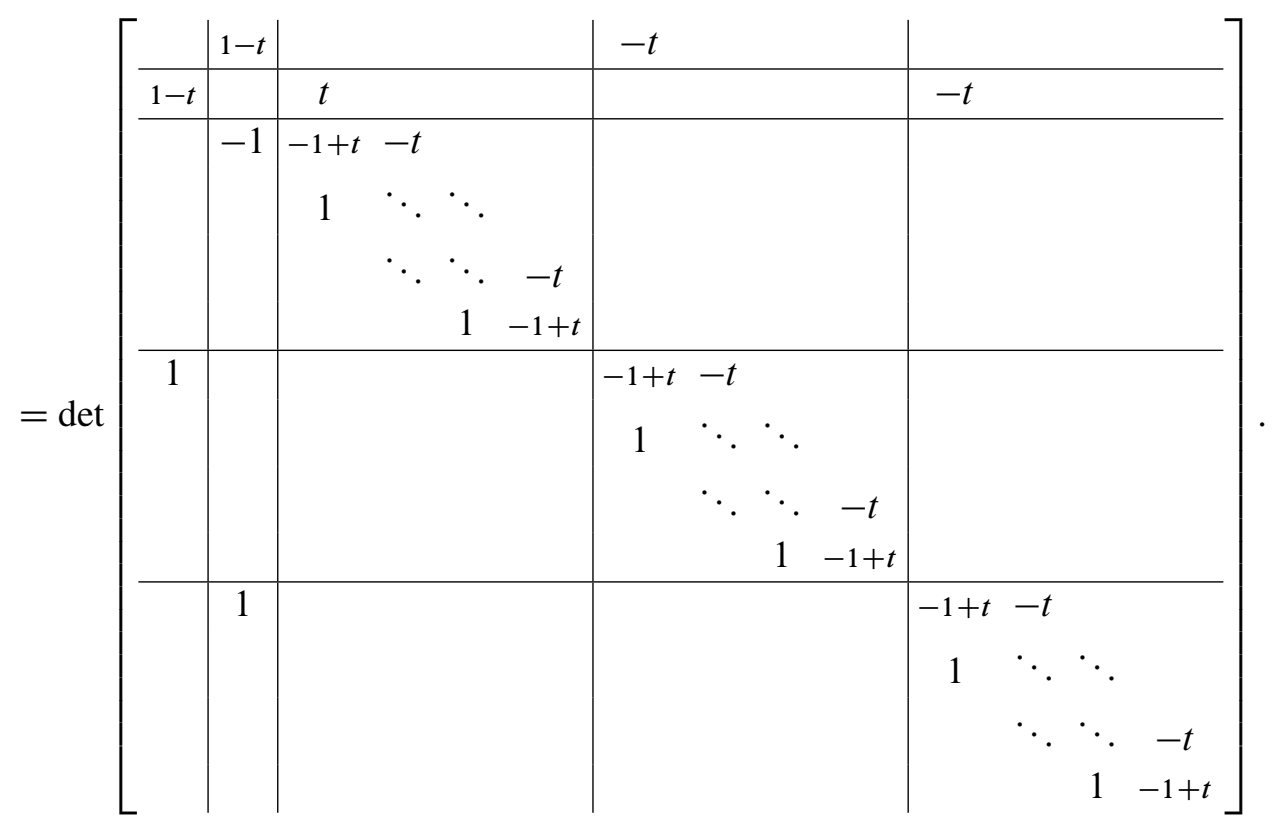

Expanding it by the $(x+1)$ th column, we have;

$$
\begin{aligned}
& \Delta_{x, y, z}(t)=(-1+t) \Delta_{x-1, y, z}(t)
\end{aligned}
$$

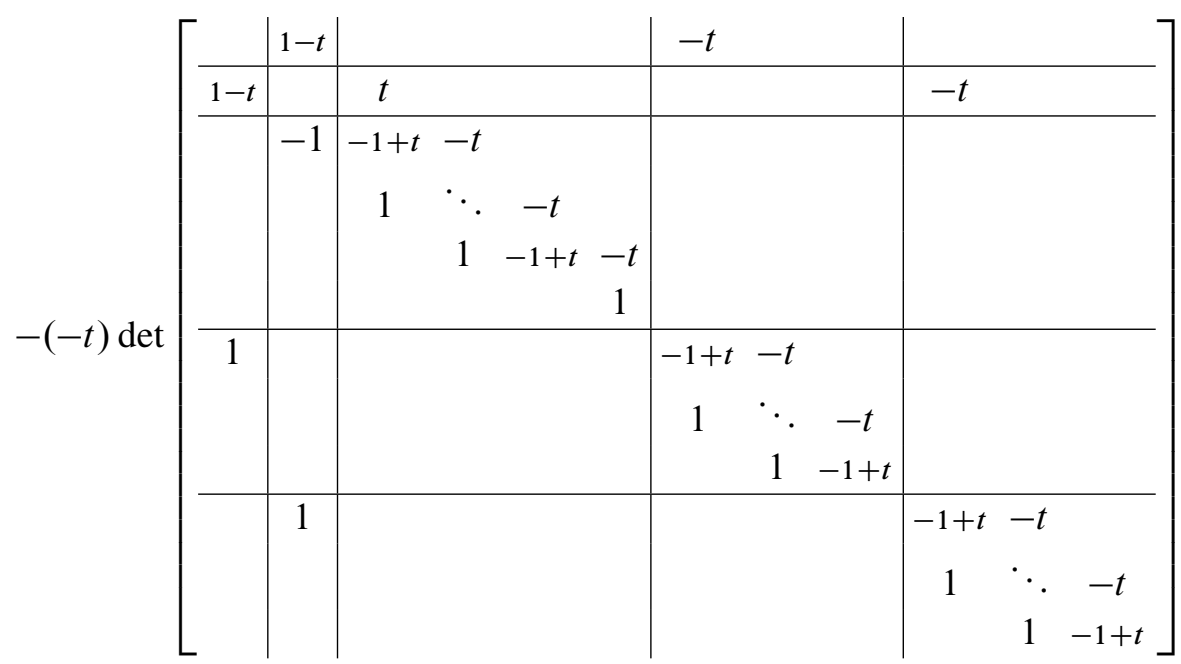

$$
\begin{aligned}
& =(-1+t) \Delta_{x-1, y, z}(t)+t \Delta_{x-2, y, z}(t) .
\end{aligned}
$$

Algebraic $8 \mathcal{G}$ Geometric Topology, Volume 6 (2006) 
If $\Delta_{i, y, z}(t)=(-1)^{i}\left(\alpha_{0}+\alpha_{1} t+\alpha_{2} t^{2}+\cdots\right)$ for $i=x-1$ and $x-2$, then

$$
\begin{aligned}
\Delta_{x, y, z}(t)= & (-1+t)(-1)^{x-1}\left(\alpha_{0}+\alpha_{1} t+\alpha_{2} t^{2}+\cdots\right) \\
& +t(-1)^{x-2}\left(\alpha_{0}+\alpha_{1} t+\alpha_{2} t^{2}+\cdots\right) \\
= & (-1)^{x}\left(\alpha_{0}+\alpha_{1} t+\alpha_{2} t^{2}+\cdots\right) .
\end{aligned}
$$

In fact, $\Delta_{x, y, z}(t)=(-1)^{x+y+z}(1-5 t+\cdots)$ for all $x, y, z \in\{2,3\}$. By induction, $\Delta_{x, y, z}(t)=(-1)^{x+y+z}(1-5 t+\cdots)$ for all $x, y, z \geq 2$.

Other cases follow by similar arguments.

Finally we are ready to prove the theorem.

Proof of Theorem 2.8 By Lemmas 2.10, 2.12, 2.14, our knot $\mathcal{K}_{2 m}$ where $(m \geq 1)$ cannot be a 3 -braid. Thus by Lemma 2.9, Theorem 2.8 follows.

\section{Uniqueness of the algebraic crossing number at minimal braid index}

\subsection{Sharpness of the MFW-inequality and conjectures}

It has been conjectured (see [8, page 357] for example) that the exponent sum in a minimal braid representation is a knot invariant.

Conjecture 3.1 (Main Conjecture) Let $\mathcal{K}$ be a knot type of braid index $b_{\mathcal{K}}$. If $K^{1}$ and $K^{2}$ are braid representatives of $\mathcal{K}$ with $b_{K^{1}}=b_{K^{2}}=b_{\mathcal{K}}$ then their algebraic crossing numbers have $c_{K^{1}}=c_{K^{2}}$.

We deform it into:

Conjecture 3.2 (Stronger Conjecture) Let $\mathcal{B}_{\mathcal{K}}$ be the set of braid representatives of $\mathcal{K}$. Let $\Phi: \mathcal{B}_{\mathcal{K}} \rightarrow \mathbb{N} \times \mathbb{Z}$ be a map such that $\Phi(K):=\left(b_{K}, c_{K}\right)$ for $K \in \mathcal{B}_{\mathcal{K}}$. Then there exists a unique $c_{\mathcal{K}} \in \mathbb{Z}$ with

$$
\Phi\left(\mathcal{B}_{\mathcal{K}}\right)=\left\{\left(b_{\mathcal{K}}+x+y, c_{\mathcal{K}}+x-y\right) \mid x, y \in \mathbb{N}\right\},
$$

a subset of the infinite quadrant region shaded in Figure 11.

The inclusion " $\supset$ " is trivial by the following argument: Let $K_{\star} \in B_{\mathcal{K}}$ be a minimal braid representative with $\Phi\left(K_{\star}\right)=\left(b_{\mathcal{K}}, c_{\mathcal{K}}\right)$. Suppose $K \in B_{\mathcal{K}}$ is obtained from $K_{\star}$ 


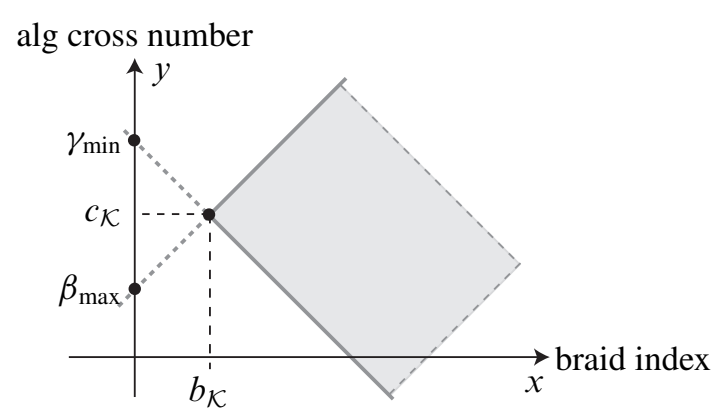

Figure 11: The region of braid representatives of $\mathcal{K}$

after applying $(+)$-stabilization $x$-times and then $(-)$-stabilization $y$-times. Then $\left(b_{\mathcal{K}}+x+y, c_{\mathcal{K}}+x-y\right)=\Phi(K) \in \Phi\left(\mathcal{B}_{\mathcal{K}}\right)$.

The MFW-inequality (2) says that $c_{K} \geq-b_{K}+\left(d_{+}+1\right), c_{K} \leq b_{K}+\left(d_{-}-1\right)$ for any $K \in \mathcal{B}_{\mathcal{K}}$. Thus

$$
\Phi\left(\mathcal{B}_{\mathcal{K}}\right) \subset\left\{(x, y) \mid b_{\mathcal{K}} \leq x,-x+\left(d_{+}+1\right) \leq y \leq x+\left(d_{-}-1\right)\right\} .
$$

Before we provide examples of the conjectures we present:

Theorem 3.3 Sharpness of the MFW-inequality implies the truth of Conjectures 3.1 and 3.2. In particular;

$$
b_{\mathcal{K}}=\frac{d_{+}-d_{-}}{2}-1, \quad c_{\mathcal{K}}=\frac{d_{+}+d_{-}}{2} .
$$

We remark that the statement in the theorem with regard to Conjecture 3.1 has been well known to many people.

Proof of Theorem 3.3 Let $K_{\star} \in \mathcal{B}_{\mathcal{K}}$ be a minimal braid representative. Since the MFW inequality (2) is sharp on $\mathcal{K}$, we have $c_{K_{\star}}-b_{\mathcal{K}}+1=d_{-}$, and $d_{+}=b_{\mathcal{K}}+c_{K_{\star}}-1$, ie, $c_{K_{\star}}=\left(d_{+}+d_{-}\right) / 2$ which is independent of the choice of $K_{\star}$. Thus we denote $c_{K_{\star}}=: c_{\mathcal{K}}$. In this case, the right side of (36) coincides with the right side of (35) and we have the other inclusion " $\subset$ " of (35).

Example 3.4 Both of the conjectures are true for unlinks, torus links, closed positive braids with a full twist (for example, the Lorenz links) [6], 2-bridge links and alternating fibered links [14], where the MFW-inequality is sharp and one can apply Theorem 3.3. 
Also Conjecture 3.1 applies to links with braid index $\leq 3$ [4]. However, this case has been settled by a completely different way, the classification of 3-braids. Namely, any link of braid index 3 admits a unique conjugacy class of 3-braid representatives or has at most two conjugacy classes of 3-braid representatives related to each other by a flype move, which does not change the algebraic crossing number of the link.

Every transversal knot $T K$ in $S^{3}$ with the standard contact structure is transversally isotopic to a transversal closed braid $K$ [1]. The Bennequin number $\beta$ is an invariant of transversal knots. By the identification of $T K$ and $K$, we have $\beta(K)=c_{K}-b_{K}$. If Conjecture 3.2 is true for $\mathcal{K}$, then the maximal Bennequin number $\beta_{\max }(\mathcal{K})$ for the knot type $\mathcal{K}$ is realized on $\mathcal{B}_{\mathcal{K}} \ni K$ 's with plotted vertices $\Phi(K)$ on the upper half boundary of the quadrant region of Figure 11. Let $\gamma(K):=c_{K}+b_{K}$. For any $K$ and its mirror image $\bar{K}$, we have $\gamma(K)=-\beta(\bar{K})$. Thus $\gamma_{\min }(\mathcal{K})=-\beta_{\max }(\overline{\mathcal{K}})$. See Figure 11. Thus, investigation of $\beta_{\max }(\mathcal{K})$ is related to Conjecture 3.2.

\subsection{Cabling and the conjectures}

In this subsection, we study behavior of the deficit of the MFW inequality under cabling and prove Theorem 3.5. As a consequence, we observe that the Conjecture 3.1 is true for many of the knots and links that appeared in Section 2, where the MFW-inequality is not sharp, ie, we cannot apply Theorem 3.3.

We also prove, in Theorems 3.10 and 3.12, that the truth of Conjecture 3.1 is "inherited" through cabling operations.

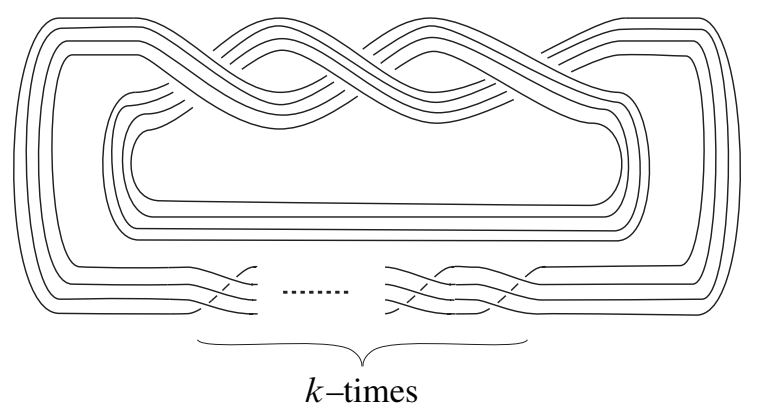

Figure 12: $(4, q)$-cable $(q=4 \cdot 3+k)$ of the right hand trefoil

Let us fix some notation. Let $\mathcal{K}$ be a knot type. Denote the $(p, q)$-cable of $\mathcal{K}$ by $\mathcal{K}_{p, q}$. Let $K$ be a braid representative with $b_{K}=b_{\mathcal{K}}$ and with algebraic crossing number $c_{K}$. Put

$$
k:=q-p \cdot c_{K}
$$

Algebraic 83 Geometric Topology, Volume 6 (2006) 
and let $K_{p, q}$ denote the $p$-parallel copies of $K$ with a $k / p$-twist (see Figure 12). We can assume that $K_{p, q}$ is on the boundary of a tubular neighborhood $N$ of $K$ (thus $K$ is the core of solid torus $N$ ). Then

$$
q=\operatorname{lk}\left(K_{p, q}, K\right)
$$

where ' $1 \mathrm{k}$ ' is the linking number. Figure 12 shows that the algebraic crossing number of $K_{p, q}$ is

$$
c_{K_{p, q}}=p^{2} c_{K}+k(p-1)=q(p-1)+p \cdot c_{K} .
$$

Thanks to [20], we know that the braid index of $\mathcal{K}_{p, q}$ satisfies

$$
b_{\mathcal{K}_{p, q}}=p \cdot b_{\mathcal{K}}
$$

Although one can see a similar result in Theorem 7 of [19], we state the following for completeness of our discussion:

Theorem 3.5 Suppose $K^{1}$ and $K^{2}$ are braid representatives of $\mathcal{K}$ with $b_{K^{1}}=b_{K^{2}}=$ $b_{\mathcal{K}}$ and with distinct algebraic crossing numbers $c_{K^{1}}<c_{K^{2}}$, (ie, Conjecture 3.1 does not apply to $\mathcal{K})$. Then the deficit $D_{\mathcal{K}_{p, q}}$ of the $M F W$-inequality for $(p, q)$-cable $\mathcal{K}_{p, q}$ is;

$$
D_{\mathcal{K}_{p, q}} \geq \frac{p}{2}\left(c_{K^{2}}-c_{K^{1}}\right) \geq p
$$

Proof of Theorem 3.5 Thanks to (38), and by the construction of $\left(K^{1}\right)_{p, q}$ and $\left(K^{2}\right)_{p, q}$, they are both minimal braid representatives of $\mathcal{K}_{p, q}$ ie, $b_{K^{1}{ }_{p, q}}=b_{K^{2}{ }_{p, q}}=$ $b_{\mathcal{K}_{p, q}}=p \cdot b_{\mathcal{K}}$.

Let $k_{1}, k_{2}$ be integers satisfying $q=p c_{K^{1}}+k_{1}=p c_{K^{2}}+k_{2}$. By (37) we have $c_{K^{1}{ }_{p, q}}=$ $p^{2} c_{K^{1}}+k_{1}(p-1)$ and $c_{K^{2}{ }_{p, q}}=p^{2} c_{K^{2}}+k_{2}(p-1)$. Therefore, $c_{K^{2}{ }_{p, q}}-c_{K^{1}{ }_{p, q}}=$ $p\left(c_{K^{2}}-c_{K^{1}}\right)$. By (2) we have $c_{K^{2}{ }_{p, q}}-b_{\mathcal{K}_{p, q}}+1 \leq d_{-} \leq d_{+} \leq c_{K^{1}{ }_{p, q}}+b_{\mathcal{K}_{p, q}}-1$, and by Definition 2.1,

$$
D_{\mathcal{K}_{p, q}} \geq \frac{1}{2}\left(c_{K^{2}{ }_{p, q}}-c_{K^{1}{ }_{p, q}}\right)=\frac{p}{2}\left(c_{K^{2}}-c_{K^{1}}\right) .
$$

This is the first inequality of (39).

Notice that $K^{1}$ and $K^{2}$ are related each other by a sequence of Markov moves [2]. Let $K^{1}=B_{1} \rightarrow B_{2} \rightarrow \cdots \rightarrow B_{n}=K^{2}$ be a Markov tower. Each arrow corresponds to either braid isotopy, stabilization or destabilization moves. Let $\left(x_{i}, y_{i}\right)$ be the braid index and the algebraic crossing number of $B_{i}$. Then $\left(x_{i+1}, y_{i+1}\right)-\left(x_{i}, y_{i}\right)=(0,0),( \pm 1, \pm 1)$ or $(\mp 1, \pm 1)$ depending on the move corresponding to the arrow between $B_{i+1}$ and 
$B_{i}$. Since $x_{1}=x_{n}=b_{\mathcal{K}}$ the difference $c_{K^{1}}-c_{K^{2}}=y_{1}-y_{n} \neq 0$ must be an even integer. Therefore, we get the second inequality of (39).

Corollary 3.6 Conjecture 3.1 is true for all $9_{42}, 9_{49}, 10_{132}, 10_{150}, 10_{156}$.

In [9], it is proved that Conjecture 3.2 also holds for the five knots.

Proof Knotscape computes that the deficit of 2 -cable $\mathcal{K}_{2,2 c_{K}+1}$ is 1 for each knot.

\begin{tabular}{|l|c|c|c|c|c|}
\hline $\mathcal{K}$ & $b_{\mathcal{K}}$ & $D_{\mathcal{K}}$ & $K$ & $c_{K}$ & $D_{\mathcal{K}_{2,2} c_{K}+1}$ \\
\hline $9_{42}$ & 4 & 1 & $a a a c B A A c B$ & 1 & 1 \\
\hline $9_{49}$ & 4 & 1 & aabbcbAbbcB & 7 & 1 \\
\hline $10_{132}$ & 4 & 2 & Abcaaa $B B B c b$ & 3 & 1 \\
\hline $10_{150}$ & 4 & 1 & aabbcbABccB & 5 & 1 \\
\hline $10_{156}$ & 4 & 1 & aaacBAAcb $A b$ & 3 & 1 \\
\hline
\end{tabular}

Comparing with (39), each $\mathcal{K}$ must have unique algebraic crossing number.

Thanks to Knotscape, the $\left(2,2 c_{\mathcal{K}_{n}}+1\right)$-cable of $\mathcal{K}_{n}=B M_{-1,-2, n, 2}$ has deficit $=1$ if $|n|$ is small. ie, Conjecture 3.1 is true for $\mathcal{K}_{n}$ if $|n|$ is small. Corollary 3.6 implies:

Corollary 3.7 Conjecture 3.1 is true for the prime links $\mathcal{A}^{n}\left(9_{42}\right)$ (see Figure 3).

Proof We know that $9_{42}$ has unique algebraic crossing number $=1$ by Corollary 3.6. Since each link component of $\mathcal{A}^{n}\left(9_{42}\right)$ is $9_{42}$, we get this corollary.

With regard to the deficit of cabled links, we conjecture that:

Conjecture 3.8 For any $q$, the limit $\lim _{p \rightarrow \infty} D_{\mathcal{K}_{p, q}}$ of deficits exists.

Remark 3.9 If Conjecture 3.8 is true, then (39) of Theorem 3.5 implies the truth of Conjecture 3.1.

We present another property of cabling:

Theorem 3.10 Let $\mathcal{K}$ be a non-trivial knot type. If Conjecture 3.1 is true for $\mathcal{K}$ then it is also true for $\mathcal{K}_{p, q}$ when $p \geq 2$.

In particular, if $c_{\mathcal{K}}$ and $c_{\mathcal{K}_{p, q}}$ denote the unique algebraic crossing numbers of $\mathcal{K}$ and $\mathcal{K}_{p, q}$ respectively in their minimal braid representatives then we have

$$
c_{\mathcal{K}_{p, q}}=(p-1) q+p \cdot c_{\mathcal{K}} .
$$


Remark 3.11 Suppose $\mathcal{K}$ is the right hand trefoil. The MFW-inequality is sharp on $\mathcal{K}$. Since its cable $\mathcal{K}_{2,7}$ has deficit $D_{\mathcal{K}_{2,7}}=1$ (see [13]), we cannot apply Theorem 3.3. However Theorem 3.10 guarantees the truth of the conjecture for $\mathcal{K}_{2,7}$.

The following proof is inspired by the work of Williams [20], whose main result can be seen in formula (38). Note that his result holds not only for cable knots but also for generalized cable links. For the sake of completeness we repeat part of his discussion.

Proof of Theorem 3.10 Assume Conjecture 3.1 is true for $\mathcal{K}$ and denote the unique algebraic crossing number at minimal braid index by $c_{\mathcal{K}}$.

Let $K$ be a braid representative of $\mathcal{K}$. Suppose $K^{\prime}$ is a braid representative of $\mathcal{K}_{p, q}$ on the boundary of a small tubular (solid torus) neighborhood $N$ of $K$. We may regard the $z$-axis as the braid axis. Let $\phi: \mathbb{R}^{3} \rightarrow \mathbb{R}^{3}$ be a diffeomorphism of compact support so that

$(\star) \quad \phi\left(K^{\prime}\right) \subset \partial \phi(N)$ has exactly $p \cdot b_{\mathcal{K}}$ maxima and $p \cdot b_{\mathcal{K}}$ minima (both nondegenerate critical points) and no other critical points and

$(\star \star)$ the "height" function $h: \partial \phi(N) \simeq T^{2} \rightarrow \mathbb{R}$ is a Morse function.

In particular, $\phi\left(K^{\prime}\right)$ has a braid position with braid index $p \cdot b_{\mathcal{K}}$.

By $(\star \star)$, a generic intersection of the horizontal plane with $\partial \phi(N) \simeq T^{2}$ consists of disjoint simple closed curves. Furthermore, these simple closed curves are either meridians of $T^{2}$ or trivial in $T^{2}$ since $\mathcal{K}$ is knotted (Remark 1 of [20]).

Remark 2 in [20] says that there is a plane $\pi$ (parallel to the $(x z)$-plane) intersecting transversely with $T^{2}$ in a meridian.

Let $J$ be an innermost one among such meridians. Then $J$ bounds a disk $d \subset \pi \cap \phi(N)$ which separates $\phi\left(K^{\prime}\right)$ into arcs $\left\{C_{i}\right\}$. Close each $C_{i}$ with aid of some arc $D_{i} \subset d$ and set $\widehat{K}_{i}:=C_{i} \cup D_{i}$. See Figure 13 .

Thanks to Remark 3 in [20], $p$ of $\widehat{K}_{i}$ 's are non-trivial (ie, do not bound any disk in $\phi(N))$ since the linking number of $J$ and $\phi\left(K^{\prime}\right)$ pushed a little bit into the interior of $\phi(N)$ is $p$.

Discard trivial $\hat{K}_{i}$ 's.

Our $\widehat{K}_{i}$ 's are not in a braid position. As in [20], we make them have a braid position: Choose another plane $\pi^{\prime}$ just below $\pi$ and call the annulus between the two planes $A$ (see Figure 13). We may assume that the other boundary curve $J^{\prime} \subset \partial A$ is parallel to $J$. 

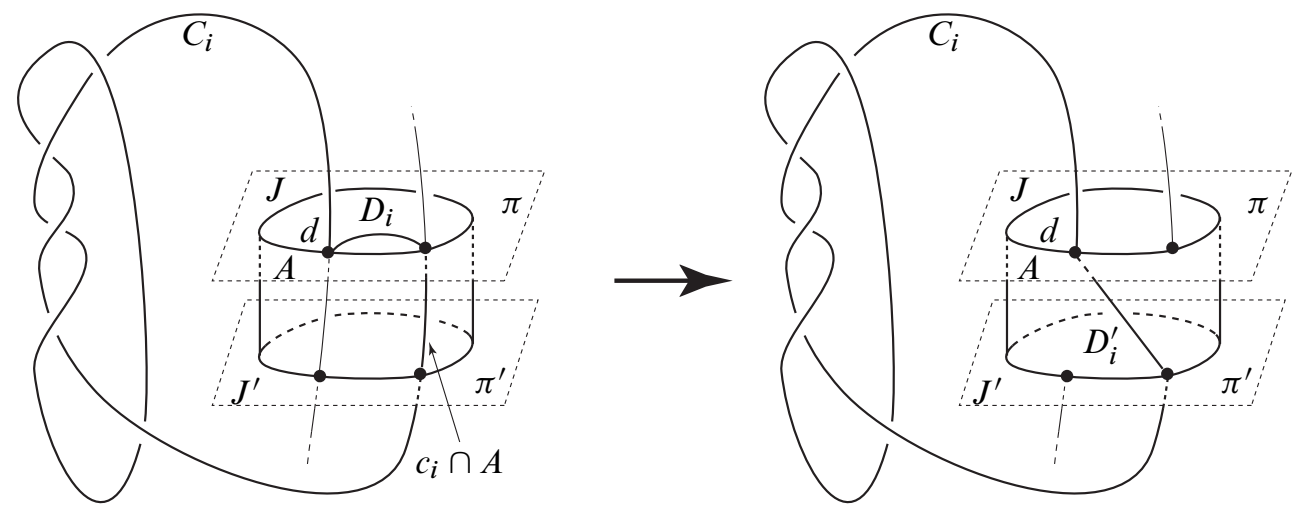

Figure 13: Construction of $K_{i}$ from $\widehat{K}_{i}$

As in the passage of Figure 13 replace the arc $D_{i} \cup\left(C_{i} \cap A\right)$ (the left sketch) with $D_{i}^{\prime} \subset A$ (the right sketch) and construct $p$-parallels;

$$
K_{i}:=\left(C_{i}-\left(C_{i} \cap A\right)\right) \cup D_{i}^{\prime} \subset \partial \phi(N) \text { for } i=1, \cdots, p,
$$

which is in a braid position. Also the $K_{i}$ 's are disjoint from each other and each is isotopic to the core of the solid torus $\phi(K) \simeq \mathcal{K}$, thus $b_{\mathcal{K}} \leq b_{K_{i}}$. Then we have

$$
\begin{aligned}
p \cdot b_{\mathcal{K}} & \leq \sum_{i=1}^{p}\left\{b_{K_{i}}=\text { number of max of } K_{i}\right\} \\
& \leq\left\{\text { number of max of } \phi\left(K^{\prime}\right)\right\}=p \cdot b_{\mathcal{K}}
\end{aligned}
$$

where the last equality holds by $(\star \star)$ above. This implies that

(†) there are no trivial $\hat{K}_{i}$ 's (we didn't have to discard anything),

$(\dagger \dagger)$ each knot has $b_{\mathcal{K}}=b_{K_{i}}$.

Let $n, 0 \leq m<p$ be integers such that

$$
q=p\left(c_{\mathcal{K}}+n\right)+m
$$

By $(\dagger)$, the $p$-component link $L:=K_{1} \cup \cdots \cup K_{p}$ is obtained from $\mathbf{K}^{\prime}:=\phi\left(K^{\prime}\right)$ by using the meridian disk $d$ to create a cutout and adding an $m / p$-twist along the annulus $A$, then gluing the end-points. See Figure 14. In other words, $L$ is the $\left(p, p\left(c_{\mathcal{K}}+n\right)\right)$-cable of $K$. From (††) we have $c_{K_{i}}=c_{K}=c_{\mathcal{K}}$. Therefore, $L$ has the algebraic crossing number 


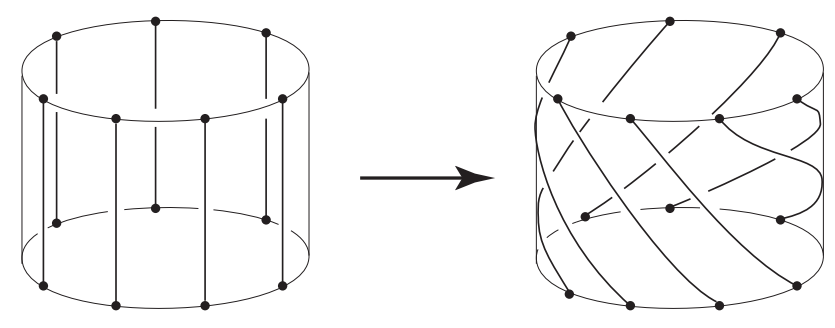

Figure 14: From $A \cap \mathbf{K}^{\prime}$ to $A \cap L$, where $p=7, m=2$

$$
\begin{aligned}
c_{L} & =\sum_{i=1}^{p} c_{K_{i}}+\sum_{i \neq j} \operatorname{lk}\left(K_{i}, K_{j}\right)=p \cdot c_{\mathcal{K}}+p(p-1)\left(c_{\mathcal{K}}+n\right) \\
& =p^{2} c_{\mathcal{K}}+p(p-1) n
\end{aligned}
$$

and $c_{\mathbf{K}^{\prime}}-c_{L}=m(p-1)$. Thus,

$$
\begin{aligned}
c_{\mathbf{K}^{\prime}} & =p^{2} c_{\mathcal{K}}+p(p-1) n+m(p-1) \\
& =p^{2} c_{\mathcal{K}}+(p-1)(p n+m) \\
& =p^{2} c_{\mathcal{K}}+\left(q-p \cdot c_{\mathcal{K}}\right) \\
& =(p-1) q+p \cdot c_{\mathcal{K}},
\end{aligned}
$$

which is independent of the choice of $\mathbf{K}^{\prime} \in \mathcal{B}_{\mathcal{K}_{p, q}}$. Compare with (37). This concludes the uniqueness of the algebraic crossing number of $\mathcal{K}_{p, q}$ at minimal braid index.

A similar result to Theorem 3.10 holds for links.

Theorem 3.12 Let $\mathcal{L}=\mathcal{K}^{(1)} \cup \cdots \cup \mathcal{K}^{(l)}$ be an $l$-component link of braid index $=b_{\mathcal{L}}$. Assume that each $\mathcal{K}^{(j)}$ is a non-trivial knot. Let $\mathcal{L}^{\prime}:=\mathcal{K}_{p, q_{1}}^{(1)} \cup \cdots \cup \mathcal{K}_{p, q_{l}}^{(l)}$ be the $p$-cable of $\mathcal{L}$ such that $q_{j}=\operatorname{lk}\left(\mathcal{K}^{(j)}, \mathcal{K}_{p, q_{j}}^{(j)}\right)$ for $j=1, \cdots, l$.

If $\mathcal{L}$ and every component $\mathcal{K}^{(j)}$ have unique algebraic crossing numbers $c_{\mathcal{L}}, c_{\mathcal{K}^{(j)}}$ in minimal braid representations, then so does $\mathcal{L}^{\prime}$.

Furthermore, let $k_{j}$ satisfy $q_{j}=p \cdot c_{\mathcal{K}^{(j)}}+k_{j}$ then

$$
c_{\mathcal{L}^{\prime}}=p^{2} c_{\mathcal{L}}+(p-1)\left(k_{1}+\cdots+k_{l}\right)
$$

Remark 3.13 The assumption for $\mathcal{K}^{(j)}$ in the second paragraph of Theorem 3.12 is essential by the following reason: If $b_{\mathcal{L}}=\sum_{j=1}^{l} b_{\mathcal{K}^{(j)}}$, then the existence of unique algebraic crossing number of $\mathcal{L}$ in minimal braid representation implies that each 
$\mathcal{K}^{(j)}$ also has unique algebraic crossing number. However, this is not true in general. For instance, assume that $\mathcal{L}=\mathcal{K}^{(1)} \cup \mathcal{K}^{(2)}$ is a 2-component link and has two braid representatives related to each other by a flype move as in Figure15. The thick gray

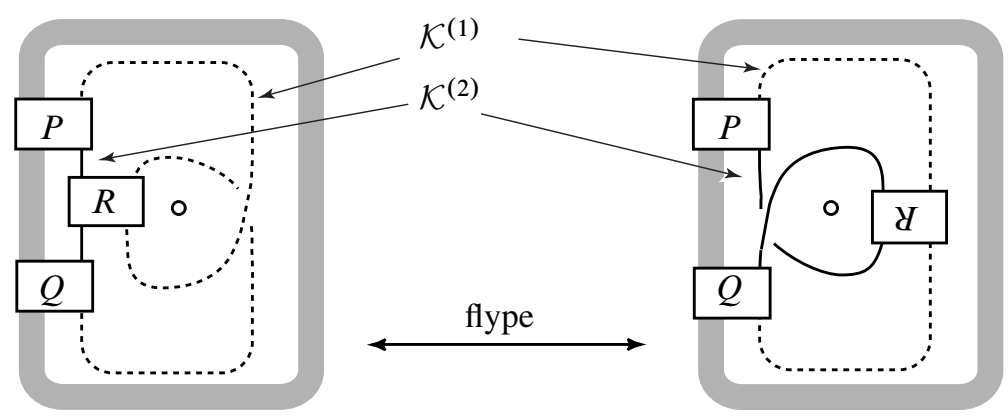

Figure 15: A flype move

arcs are parallel braid strands of $\mathcal{L}$. Braidings occur inside the boxes $P, Q, R$. In particular, box $R$ contains even number of half twists of $\mathcal{K}^{(1)}$ (dashed arc) and $\mathcal{K}^{(2)}$ (black arc). The flype move preserves the number of braid strands and the algebraic crossing number of the link, but it changes the algebraic crossing numbers of link components $\mathcal{K}^{(1)}, \mathcal{K}^{(2)}$. Namely, in the passage from the left sketch to the right sketch, the algebraic crossing number of $\mathcal{K}^{(1)}$ decreases by 1 and the one for $\mathcal{K}^{(2)}$ increases by 1 . (This means that a flype move in general cannot be a composition of exchange moves.)

Proof of Theorem 3.12 Suppose $L=K^{(1)} \cup \cdots \cup K^{(l)}$ is a minimal braid representative of $\mathcal{L}=\mathcal{K}^{(1)} \cup \cdots \cup \mathcal{K}^{(l)}$, ie, $b_{L}=b_{\mathcal{L}}$. Let $c_{x, y}:=2 \cdot \operatorname{lk}\left(\mathcal{K}^{(x)}, \mathcal{K}^{(y)}\right)$ for $x \neq y$. Then

$$
c_{\mathcal{L}}=c_{L}=\sum_{1 \leq x<y \leq l} c_{x, y}+\sum_{j=1}^{l} c_{\mathcal{K}^{(j)}} .
$$

Let $k_{j}, n_{j}, 0 \leq m_{j}<p$ be integers with

$$
q_{j}=p \cdot c_{\mathcal{K}^{(j)}}+k_{j}=p\left(c_{\mathcal{K}^{(j)}}+n_{j}\right)+m_{j} \quad \text { for } j=1, \cdots, l .
$$

Williams proved that the braid index of $\mathcal{L}^{\prime}=\mathcal{K}_{p, q_{1}}^{(1)} \cup \cdots \cup \mathcal{K}_{p, q_{l}}^{(l)}$ is $p \cdot b_{\mathcal{L}}$ [20]. Let $L^{\prime}$ be a minimal braid representative of $\mathcal{L}^{\prime}$. Let $N_{j}$ be a tubular neighborhood of $K^{(j)}$. Let $\phi$ be a compact support diffeomorphism of $\mathbb{R}^{3}$ such that $\phi\left(L^{\prime}\right)=: K^{\prime(1)} \cup \cdots \cup K^{\prime(l)} \subset$ $\partial \phi\left(N_{1} \cup \cdots N_{l}\right)$ has a minimal braid position. 
As we did in (41), for each $j=1, \cdots, l$, construct $p$ parallels $K_{1}^{(j)}, \cdots, K_{p}^{(j)} \subset$ $\partial \phi\left(N_{j}\right)$ from each $K^{\prime(j)}$ by cutting out an inner most meridian disk $d_{j} \subset \phi\left(N_{j}\right)$ and adding an $m_{j} / p$-twist along annulus $A_{j} \subset \partial \phi\left(N_{j}\right)$ then gluing. Thus,

$$
\operatorname{lk}\left(K_{i}^{(j)}, K^{(h)}\right)= \begin{cases}c_{\mathcal{K}^{(j)}}+n_{j} & \text { when } j=h, \\ \operatorname{lk}\left(K^{(j)}, K^{(h)}\right)=\frac{1}{2} c_{j, h} & \text { otherwise. }\end{cases}
$$

Let

$$
L_{i}:=K_{i}^{(1)} \cup \cdots \cup=K_{i}^{(l)} \text { for } i=1, \cdots, p .
$$

Thanks to [20] we know that $L_{i} \simeq L$ and $b_{L_{i}}=b_{L}=b_{\mathcal{L}}$. By assumption of Theorem 3.12 , it follows that $c_{L_{i}}=c_{L}=c_{\mathcal{L}}$. The $(p \cdot l)$-component link $L_{1} \cup \cdots \cup L_{p}$ has the algebraic crossing number;

$$
\begin{aligned}
c_{L_{1} \cup \cdots \cup L_{p}} & =\sum_{i=1}^{p} c_{L_{i}}+\sum_{x \neq y} \operatorname{lk}\left(L_{x}, L_{y}\right) \\
& =p \cdot c_{\mathcal{L}}+\sum_{i=1}^{p}(p-1) \operatorname{lk}\left(L_{i}, L\right) \\
& =p \cdot c_{\mathcal{L}}+\sum_{i=1}^{p}(p-1)\left\{\sum_{j=1}^{l} \operatorname{lk}\left(K_{i}^{(j)}, K^{(j)}\right)+\sum_{x \neq y} 1 \mathrm{k}\left(K_{i}^{(x)}, K^{(y)}\right)\right\} \\
& \stackrel{(45)}{=} p \cdot c_{\mathcal{L}}+p(p-1)\left\{\sum_{j=1}^{l}\left(c_{\mathcal{K}^{(j)}}+n_{j}\right)+\sum_{x<y} c_{x, y}\right\} \\
& \stackrel{(43)}{=} p \cdot c_{\mathcal{L}}+p(p-1)\left(c_{\mathcal{L}}+\sum_{j=1}^{l} n_{j}\right) \\
& =p^{2} c_{\mathcal{L}}+p(p-1)\left(\sum_{j=1}^{l} n_{j}\right) .
\end{aligned}
$$

Since only the difference between $L_{1} \cup \cdots \cup L_{p}$ and $L^{\prime}$ occurs on the annuli $A_{1}, \cdots, A_{l}$, we have

$$
\begin{aligned}
c_{L^{\prime}} & =c_{L_{1} \cup \cdots \cup L_{p}}+(p-1) \sum_{j=1}^{l} m_{j} \\
& =p^{2} c_{\mathcal{L}}+(p-1) \sum_{j=1}^{l}\left(p n_{j}+m_{j}\right)
\end{aligned}
$$

Algebraic $8 \mathcal{G}$ Geometric Topology, Volume 6 (2006) 


$$
\stackrel{(44)}{=} p^{2} c_{\mathcal{L}}+(p-1) \sum_{j=1}^{l} k_{j},
$$

which is independent of the choice of braid representative $L^{\prime}$.

With regard to Conjecture 3.2 we have:

Theorem 3.14 Let $\mathcal{L}=\mathcal{K}^{(1)} \cup \cdots \cup \mathcal{K}^{(l)}$ be an $l$-component link satisfying all the assumptions in Theorem 3.12. If Conjecture 3.2 is true for $\mathcal{L}$ then it is also true for its $p$-cable $\mathcal{L}^{\prime}:=\mathcal{K}_{p, q_{1}}^{(1)} \cup \cdots \cup \mathcal{K}_{p, q_{l}}^{(l)}$.

Proof of Theorem 3.14 Let $L$ (resp. $L^{\prime}$ ) be a braid representative of $\mathcal{L}$ (resp. $\mathcal{L}^{\prime}$ ). Take tubular neighborhoods $N=N_{1} \cup \cdots \cup N_{l}$ of $L$ (each $N_{j}$ is a solid torus) and let $\phi: \mathbb{R}^{3} \rightarrow \mathbb{R}^{3}$ be a compact support diffeo morphism such that $\phi(L)=: K^{(1)} \cup \cdots \cup K^{(l)}$, $\phi\left(L^{\prime}\right)=: K^{\prime(1)} \cup \cdots \cup K^{\prime(l)}$ have braid positions. They are not necessarily minimal braid representatives and in general $b_{\phi\left(L^{\prime}\right)} \neq p \cdot b_{\phi(L)}$. We may assume that $K^{\prime(j)} \subset$ $\partial \phi\left(N_{j}\right) \simeq T^{2}$.

Let plane $\pi_{j}=\left\{(x, y, z) \mid y=y_{0}\right\}$, innermost meridian loop $J_{j} \subset \pi_{j} \cap \partial \phi\left(N_{j}\right)$, and meridian disk $d_{j} \subset \pi_{j} \cap \phi\left(N_{j}\right)$ be as in the proof of Theorem 3.10. We may assume that the braid axis is not contained in $\pi_{j}$ ie, $y_{0} \neq 0$.

We deform $K^{\prime(j)}$ in the following way: Suppose sub-arcs $u \subset K^{\prime(j)}$ and $v \subset J_{j}$ bound a disk $\mathcal{D} \subset \partial \phi\left(N_{j}\right) \simeq T^{2}$. If $\mathcal{D}$ is innermost, then replace $u$ with $v$. Repeat this until $K^{\prime(j)}$ and $J_{j}$ do not bound any disk in $T^{2}$. Add up all the linking numbers of $\partial \mathcal{D}$ 's with the $z$-axis and denote it by $x^{(j)} \geq 0$.

Next, from the deformed $K^{\prime(j)}$ above, construct $p$-parallels $K_{1}^{\prime(j)}, \cdots, K_{p}^{\prime(j)}$ as in (41). Since the plane $\pi_{j}$ does not contain the $z$-axis, the $m_{j} / p$-twist along a thin annulus does not change the number of braid strands.

Suppose that $b_{K^{(j)}}=b_{\mathcal{K}^{(j)}}+y^{(j)}$ and $b_{K_{i}^{\prime(j)}}=b_{\mathcal{K}^{(j)}}+y^{(j)}+z_{i}^{(j)}$ with $y^{(j)}, y^{(j)}+$ $z_{i}^{(j)} \geq 0$. Let $L_{i}:=K_{i}^{(1)} \cup \cdots \cup=K_{i}^{(l)}$ then

$$
\begin{gathered}
b_{L_{i}}=\sum_{j=1}^{l} b_{K_{i}^{(j)}}=b_{\mathcal{L}}+\sum_{j=1}^{l}\left(y^{(j)}+z_{i}^{(j)}\right), \\
b_{\phi\left(L^{\prime}\right)}=\sum_{i=1}^{p} b_{L_{i}}+\sum_{j=1}^{l} x^{(j)} \stackrel{(46)}{=} p \cdot b_{\mathcal{L}}+\sum_{j=1}^{l}\left(x^{(j)}+p \cdot y^{(j)}+\sum_{i=1}^{p} z_{i}^{(j)}\right) .
\end{gathered}
$$


Since $L_{i} \simeq \mathcal{L}$, our assumption of this theorem and (46) give us

$$
c_{\mathcal{L}}-\sum_{j=1}^{l}\left(y^{(j)}+z_{i}^{(j)}\right) \leq c_{L_{i}} \leq c_{\mathcal{L}}+\sum_{j=1}^{l}\left(y^{(j)}+z_{i}^{(j)}\right) .
$$

As in the proof of Theorem 3.12, let $k_{j}, n_{j}, 0 \leq m_{j}<p$ satisfy $q_{j}=p \cdot c_{\mathcal{K}^{(j)}}+k_{j}=$ $p\left(c_{\mathcal{K}^{(j)}}+n_{j}\right)+m_{j}$. Then we have

$$
\begin{aligned}
c_{L_{1} \cup \ldots \cup L_{p}} & =\sum_{i=1}^{p} c_{L_{i}}+\sum_{x \neq y} \operatorname{lk}\left(L_{x}, L_{y}\right) \\
& \stackrel{(48)}{\leq} p\left(c_{\mathcal{L}}+\sum_{j=1}^{l} y^{(j)}\right)+\sum_{i=1}^{p} \sum_{j=1}^{l} z_{i}^{(j)}+p(p-1)\left(c_{\mathcal{L}}+\sum_{j=1}^{l} n_{j}\right) \\
& =p^{2} c_{\mathcal{L}}+p(p-1)\left(\sum_{j=1}^{l} n_{j}\right)+\sum_{j=1}^{l}\left(p \cdot y^{(j)}+\sum_{i=1}^{p} z_{i}^{(j)}\right),
\end{aligned}
$$

and

$$
\begin{aligned}
c_{\phi\left(L^{\prime}\right)} & \leq c_{L_{1} \cup \ldots \cup L_{p}}+\sum_{j=1}^{l} m_{j}+\sum_{j=1}^{l} x^{(j)} \\
& \leq p^{2} c_{\mathcal{L}}+(p-1) \sum_{j=1}^{l} k_{j}+\sum_{j=1}^{l}\left(x^{(j)}+p \cdot y^{(j)}+\sum_{i=1}^{p} z_{i}^{(j)}\right) .
\end{aligned}
$$

Similarly,

$$
p^{2} c_{\mathcal{L}}+(p-1) \sum_{j=1}^{l} k_{j}-\sum_{j=1}^{l}\left(x^{(j)}+p \cdot y^{(j)}+\sum_{i=1}^{p} z_{i}^{(j)}\right) \leq c_{\phi\left(L^{\prime}\right)} .
$$

We conclude the theorem by (42), (47), (49) and (50).

Corollary 3.15 Conjectures 3.1 and 3.2 apply to iterated torus knots.

Proof of Corollary 3.15 We know that the both conjectures apply to torus knots (Example 3.4). Thanks to Theorems 3.10, 3.12 and 3.14, we have this corollary.

\subsection{Connect sum and the conjecture}

We will prove the following:

Algebraic 8 Geometric Topology, Volume 6 (2006) 
Theorem 3.16 If Conjecture 3.1 is true for knot types $\mathcal{K}^{1}$ and $\mathcal{K}^{2}$ then it is also true for the connect sum $\mathcal{K}^{1} \sharp \mathcal{K}^{2}$.

In particular, denoting the unique algebraic crossing numbers of $\mathcal{K}^{i}$ in minimal braid representatives by $c_{\mathcal{K}^{i}}$ we have

$$
c_{\mathcal{K}^{1} \sharp \mathcal{K}^{2}}=c_{\mathcal{K}^{1}}+c_{\mathcal{K}^{2}}
$$

Before we prove Theorem 3.16 let us recall two important known results:

Lemma 3.17 [10, Theorem 2.12] Up to ordering of summands, there is a unique expression for a knot type $\mathcal{K}$ as a finite connect sum of prime knots.

Lemma 3.18 (The composite braid theorem, [3].) Let $\mathcal{K}$ be a composite link, and let $K$ be an arbitrary closed $n$-braid representative of $\mathcal{K}$. Then there is an obvious composite $n$-braid representative $K^{\bullet}$ of $\mathcal{K}$ (see Figure 16) and a finite sequence of closed $n$-braids:

$$
K=K_{0} \rightarrow K_{1} \rightarrow \cdots \rightarrow K_{m}=K^{\bullet}
$$

such that $K_{i+1}$ is obtained from $K_{i}$ by either braid isotopy or an exchange move.

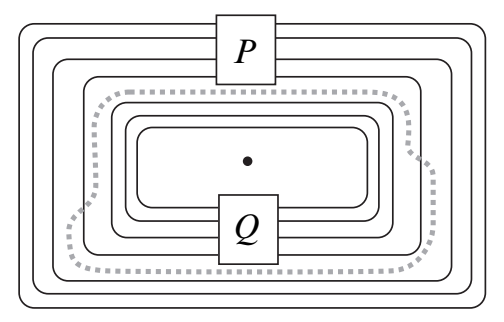

Figure 16: An obvious composite braid

Proof of Theorem 3.16 Since an exchange move does not change the algebraic crossing number, Lemmas 3.17 and 3.18 imply the truth of Theorem 3.16.

As a corollary of Theorem 3.16 we have:

Theorem 3.19 If Conjecture 3.2 is true for $\mathcal{K}^{1}, \mathcal{K}^{2}$ then it is also true for $\mathcal{K}^{1} \sharp \mathcal{K}^{2}$. 
Proof of Theorem 3.19 Let $K$ be a braid representative of $\mathcal{K}^{1} \sharp \mathcal{K}^{2}$. By Lemma 3.18, after applying exchange moves and braid isotopy to $K$ one can get a composite braid representative $K^{\bullet}=K^{1} \sharp K^{2}$. Suppose $b_{K^{i}}=b_{\mathcal{K}^{i}}+x_{i}$ with $x_{i} \geq 0$. Then

$$
\begin{aligned}
b_{K} & =b_{K}=b_{K^{1}}+b_{K^{2}}-1=\left(b_{\mathcal{K}^{1}}+b_{\mathcal{K}^{2}}-1\right)+\left(x_{1}+x_{2}\right) \\
& =b_{\mathcal{K}^{1} \sharp \mathcal{K}^{2}}+\left(x_{1}+x_{2}\right) .
\end{aligned}
$$

Our assumption gives $c_{\mathcal{K}^{i}}-x_{i} \leq c_{K^{i}} \leq c_{\mathcal{K}^{i}}+x_{i}$. Since $c_{K}=c_{K} \bullet=c_{K^{1}}+c_{K^{2}}$, we have $\left(c_{\mathcal{K}^{1}}+c_{\mathcal{K}^{2}}\right)-\left(x_{1}+x_{2}\right) \leq c_{K} \leq\left(c_{\mathcal{K}^{1}}+c_{\mathcal{K}^{2}}\right)+\left(x_{1}+x_{2}\right)$. Thanks to Theorem 3.16 ,

$$
c_{\mathcal{K}^{1} \sharp \mathcal{K}^{2}}-\left(x_{1}+x_{2}\right) \leq c_{K} \leq c_{\mathcal{K}^{1} \sharp \mathcal{K}^{2}}+\left(x_{1}+x_{2}\right) .
$$

The truth of Conjecture 3.2 follows by (51), (52).

\section{References}

[1] D Bennequin, Entrelacements et équations de Pfaff, from: "Third Schnepfenried geometry conference, Vol. 1 (Schnepfenried, 1982)”, Astérisque 107, Soc. Math. France, Paris (1983) 87-161 MR753131

[2] J S Birman, Braids, links, and mapping class groups, Annals of Mathematics Studies 82, Princeton University Press (1974) MR0375281

[3] J S Birman, W W Menasco, Studying links via closed braids. IV. Composite links and split links, Invent. Math. 102 (1990) 115-139 MR1069243

[4] J S Birman, W W Menasco, Studying links via closed braids. III. Classifying links which are closed 3-braids, Pacific J. Math. 161 (1993) 25-113 MR1237139

[5] J S Birman, W W Menasco, Stabilization in the braid groups. I. MTWS, Geom. Topol. 10 (2006) 413-540 MR2224463

[6] J Franks, R F Williams, Braids and the Jones polynomial, Trans. Amer. Math. Soc. 303 (1987) 97-108 MR896009

[7] E Giroux, Géométrie de contact: de la dimension trois vers les dimensions supérieures, from: "Proceedings of the International Congress of Mathematicians, Vol. II (Beijing, 2002)”, Higher Ed. Press, Beijing (2002) 405-414 MR1957051

[8] V F R Jones, Hecke algebra representations of braid groups and link polynomials, Ann. of Math. (2) 126 (1987) 335-388 MR908150

[9] K Kawamuro, Conjectures on the braid index and the algebraic crossing number, to appear in proceedings of "Intelligence of Low Dimensional Topology (Hiroshima, 2006)"

[10] W B R Lickorish, An introduction to knot theory, Graduate Texts in Mathematics 175, Springer (1997) MR1472978 
[11] P M Melvin, H R Morton, Fibred knots of genus 2 formed by plumbing Hopf bands, J. London Math. Soc. (2) 34 (1986) 159-168 MR859157

[12] H R Morton, Seifert circles and knot polynomials, Math. Proc. Cambridge Philos. Soc. 99 (1986) 107-109 MR809504

[13] H R Morton, H B Short, The 2-variable polynomial of cable knots, Math. Proc. Cambridge Philos. Soc. 101 (1987) 267-278 MR870598

[14] K Murasugi, On the braid index of alternating links, Trans. Amer. Math. Soc. 326 (1991) 237-260 MR1000333

[15] W D Neumann, Private communication

[16] W D Neumann, L Rudolph, Difference index of vectorfields and the enhanced Milnor number, Topology 29 (1990) 83-100 MR1046626

[17] W D Neumann, D Zagier, Volumes of hyperbolic three-manifolds, Topology 24 (1985) 307-332 MR815482

[18] A Stoimenow, Properties of closed 3-braids arXiv:math.GT/0606435

[19] A Stoimenow, On the crossing number of positive knots and braids and braid index criteria of Jones and Morton-Williams-Franks, Trans. Amer. Math. Soc. 354 (2002) 3927-3954 MR1926860

[20] R F Williams, The braid index of generalized cables, Pacific J. Math. 155 (1992) 369-375 MR1178031

[21] P Xu, The genus of closed 3-braids, J. Knot Theory Ramifications 1 (1992) 303-326 MR1180404

Math Department, Rice University, 6100 S Main St

Houston TX 77005-1892, USA

keiko.kawamuro@rice.edu

Received: 28 April 2006 